\title{
ROBUST CONTROL OF REDUNDANTLY ACTUATED DYNAMICAL SYSTEMS
}

\author{
A Thesis \\ by \\ MANORANJAN MAJJI
}

\begin{abstract}
Submitted to the Office of Graduate Studies of Texas A\&M University in partial fulfillment of the requirements for the degree of

MASTER OF SCIENCE
\end{abstract}

May 2006

Major Subject: Aerospace Engineering 


\title{
ROBUST CONTROL OF REDUNDANTLY ACTUATED DYNAMICAL SYSTEMS
}

\author{
A Thesis \\ by \\ MANORANJAN MAJJI
Submitted to the Office of Graduate Studies of Texas A\&M University
in partial fulfillment of the requirements
for the degree of
MASTER OF SCIENCE

Approved by:

Chair of Committee, John L. Junkins

Committee Members, Srinivas R. Vadali

Aniruddha Datta

Othon K. Rediniotis

Head of Department, Helen L. Reed

May 2006

Major Subject: Aerospace Engineering 


\begin{abstract}
Robust Control of Redundantly Actuated Dynamical Systems. (May 2006) Manoranjan Majji, B.E. (Hons), Birla Institute of Technology and Science Chair of Advisory Committee: Dr. John L. Junkins
\end{abstract}

The eigenstructure assignment scheme for robust multivariable feedback control is extended to redundantly actuated dynamical systems. It is shown that an orthonormal set of close loop eigenvectors is always exactly assignable in the case of redundant actuation proving the inherent robustness in the control design methodology. A choice of close loop eigenvector set to minimize the feedback gain matrix is suggested. Partial Eigenstructure Assignment methodology is proposed for second order mechanical systems. A methodology for coordinated actuation of redundant actuator sets by a trained weighted minimum norm solution is presented. To apply the methodology to hyper-redundant actuator arrays, for application to smart actuator arrays, a novel adaptive discretization algorithm is proposed. The adaptive aggregation strategy, based on the physics of the system, introduces nodes, to optimize a performance index of the overall plant model. The dimensionality of the inputs thus reduces to a finite number, making it a candidate plant for control by the robust redundant control scheme. The adaptive aggregation together with robust redundant control methodology is demonstrated on a finite element model of a novel morphing wing. This schema unifies the traditionally disparate methods of modeling and controller design. 
To Goddess Saraswathi, Amma and Daddy 


\section{ACKNOWLEDGMENTS}

My deepest appreciation to my advisor, Dr. John L. Junkins for making these results possible. I am highly indebted to Dr. Junkins for persistently exciting my learning modes and having extended his invaluable guidance, encouragement, financial support and kindling my interest in various spheres of dynamics and control through hours of thought provoking discussions.

Many thanks to Dr. Srinivas R. Vadali for motivating discussions in and out of the class room and having kindly agreed to serve on my committee.

I am grateful to Dr. Othon K. Rediniotis for providing me invaluable experience with experiments. Thanks are also due to Dr. Aniruddha Datta for serving on my committee.

Thanks to Drs. Lance W. Traub and P. Singla for helping me out with fine aspects of research.

Rajnish, Bong Su, and Pravin have made my student life highly enjoyable! Thanks a lot and I look forward to more fun!

Thanks to Ms. Lisa Willingham and Ms. Karen Knabe for helping out with office work.

I am grateful to my parents, for the constant encouragement, love and affection they have bestowed on to me. Sai akka Cinnadodda and GB anna have also been a source of great support. Many thanks to them!!! 
The support of the Texas Institute for Intelligent Bio-Nano Materials and Structures for Aerospace Vehicles, funded by NASA Cooperative Agreement No. NCC-102038 is acknowledged. Any opinions, findings and conclusions or recommendations expressed in this material are those of the author and do not necessarily reflect the views of the National Aeronautics and Space Administration. 


\section{TABLE OF CONTENTS}

INTRODUCTION . . . . . . . . . . . . . . . . . . . . . . . 1

A. Redundancy in Mechanics . . . . . . . . . . . . . . . 1

1. Kinematic Redundancy . . . . . . . . . . . . . . . . 1

2. Actuator Redundancy . . . . . . . . . . . . . . . 2

B. Actuator Redundancy Management . . . . . . . . . . . 3

1. Robotic Applications . . . . . . . . . . . . . 3

2. Redundant Actuation in Aircraft . . . . . . . . . . 4

3. Spacecraft Applications . . . . . . . . . . . . 4

C. Redundantly Actuated Dynamical Systems . . . . . . . . 5

1. Controllability .............. . . 6

2. Other Features . . . . . . . . . . . . . 7

D. Organization of the Thesis . . . . . . . . . 7

II EIGENSTRUCTURE ASSIGNMENT . . . . . . . . . . . . . 9

A. Eigenstructure Assignment Methodology . . . . . . . . . . 10

1. Robustness .................. . 11

2. Assignability of Eigenvectors . . . . . . . . . . 12

B. Robust Eigenstructure Assignment (EsA) for Redundantly Actuated Systems . . . . . . . . . . . . . . 13

1. Target Sets of Eigenvectors . . . . . . . . . . . . . 16

C. Second Order Mechanical Systems: Partial Eigenstructure Assignment . . . . . . . . . . . . . . . . . . 18

1. Partial Eigenstructure Assignment of Second Order Systems . . . . . . . . . . . . . . . . 19

2. Example ................. . . 21

3. Features of the Partial EsA Algorithm . . . . . . . . 22

III EXAMPLE APPLICATION . . . . . . . . . . . 24

IV COORDINATION OF REDUNDANT ACTUATOR SETS . . . 30

A. Trained Weighting Approach for Coordination . . . . . . 30

V APPLICATION TO DISTRIBUTED CONTROL PROBLEMS: HYPER-REDUNDANT ACTUATION . . . . . . . . . . . 34 
A. Algorithm for Adaptive Discretization . . . . . . . . . . 35

B. Application to the Morphing Wing . . . . . . . . . 36

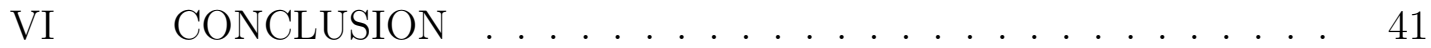

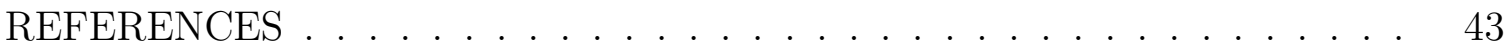

APPENDIX A . . . . . . . . . . . . . . . . . . . . . . . 49

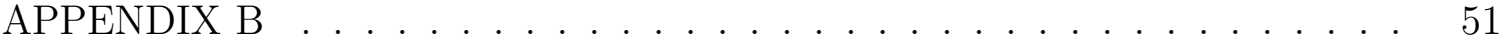

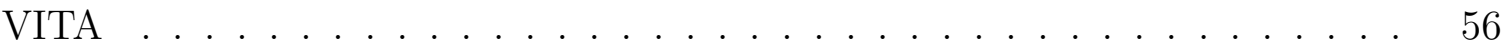




\section{LIST OF TABLES}

TABLE $\quad$ Page

I $\quad$ Applied additive parameter perturbations to plant model . . . . . . . 28

II Eigenvalues for the structural model of the morphing wing . . . . . . 40 


\section{LIST OF FIGURES}

FIGURE

Page

$1 \quad$ Eigenstructure assignment: $2 \mathrm{D}$ example . . . . . . . . . . . . 16

2 Comparison of various orthonormal target sets for feedback gain minimization ...................... 18

3 Schematic of a redundantly actuated robot . . . . . . . . . . 24

4 Robust position tracking of the platform coordinates . . . . . . . 28

5 Robust velocity tracking of the platform coordinates . . . . . . . . 29

6 Schematic illustrating the idea of training the weight matrix approximating effectiveness . . . . . . . . . . . . . . 32

$7 \quad$ Control inputs on payload with and without trained weighting . . . . 33

8 Control inputs on arm and shoulder joints with and without trained

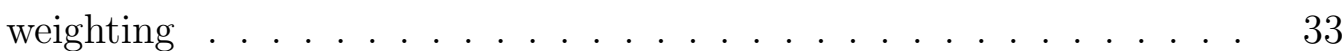

$9 \quad$ Schematic of the adaptive aggregation scheme for hyper-redundant actuation ........................ 36

10 Aggregation history with Hamdan Nayfeh Greedy strategy . . . . . . 37

11 Conditioning of the control influence matrix with mode addition . . . 38

12 Morphing Wing experiment . . . . . . . . . . . . 39

13 Tracking control law with adaptive local linearization scheme . . . . 54

14 Tracking control with $20 \%$ perturbed initial conditions . . . . . . . 55

15 Schematic of the two link rigid manipulator . . . . . . . . . . . 55 


\section{CHAPTER I}

\section{INTRODUCTION}

Redundancy in sensing and actuation has taken a center stage in control of modern day dynamical systems, owing to their complex functionality and increased performance targets. Classical control theory being largely developed for under-actuated systems, there is a need to extend the domain of control theory and develop methods to handle redundancy effectively.

\section{A. Redundancy in Mechanics}

The current machinery for dealing with redundancy issues can be broadly classified in to the following categories.

- Kinematic Redundancy

- Actuator Redundancy

This classification is entirely based on the nature of the problem (Inspired from Nakamura [1], the classification here is required to focus the features of the present thesis). In this thesis, we are only interested in developing methods to handle actuator redundancy. Let us take a close look at each of the types.

\section{Kinematic Redundancy}

This type of redundancy is clearly understood with examples from robotics. Consider a robotic arm moving in a 2-D plane. If there are obstacles in the plane, it would need more than two joints for obstacle avoidance and dexterous end effector manipulation. Kinematic redundancy constitutes problems of this nature. In the 
robotics literature, this dimension of the manipulation variable (number of independent generalized coordinates) is called the degree of Manipulability and the number of extra joints (the redundant coordinates given by the constraints), the degree of Redundancy. When the constraint equations are known, methods of analytical dynamics are typically used to solve the system equations in terms of the generalized coordinates as summarized by Junkins and Turner [2]. Constrained optimization methods [1] are routinely used in solving these problems. When the redundancy is small, singular configurations occur quite often, whose measures and avoidance schemes are widely known $[1,3]$. When kinematic redundancies are large, Chirakjian[4], presents a continuum type methodology for determining kinematically optimal configurations.

\section{Actuator Redundancy}

Actuator redundancy is the scenario where in we have more actuators than the degrees of freedom of the system to be controlled. In most cases, this occurs together with kinematic redundancy (passive damping at each kinematic degree of freedom is an example). In many cases, the actuators themselves are dynamical systems that may contribute additional degrees of freedom to be considered. The proposed thesis focuses on issues dealing with this type of redundancy. While the theoretical kinematic redundancy reduces the number of generalized coordinates in the mathematical model of the system, the redundant actuator sets act as generalized external forces in the equations of motion influencing the much smaller number of states. Physically this is found in robotic systems when the external forces act on the redundant kinematic degrees of freedom. A three degree of freedom planar manipulator with with motors at all three joints is an example. There have been several ways of redundancy handling in the literature. 
This thesis proposes a methodology to handle actuator redundancy at a level chosen by the designer. It is strongly felt that redundancy in actuation can be utilized to provide robustness and coordination in systems, going one step beyond the conventional control system objectives of stability, regulation or tracking. Redundancy in actuation can be used to increase the system reliability (performance under actuator failure) and the load distributions can be optimized to meet certain performance criteria [5]. In lieu of the importance of redundant actuation, let us briefly look at certain ways of handling redundancy in use in several applications.

\section{B. Actuator Redundancy Management}

Redundancy in actuation has applications and methods of management in most areas of engineering. This section discusses some of these areas and points at the corresponding methods of handling redundancy, particular to that area. There is a heavy bias towards applications in mechanical systems.

\section{Robotic Applications}

In robotic systems, redundancy is handled by several strategies. One particular approach shown in Nakamura [1] uses a prioritization approach achieving local/global optimal control formulations. Typical applications include robots with parallel actuated wrists and dexterous fingers. Modeling of such closed link robot mechanisms with redundant actuators poses an equally difficult challenge. The advantages of redundancy in handling actuator failure and in optimizing load distribution were pointed out by Nakamura and Ghodoussi[5]. They suggested modeling of redundant joints by equivalent open chains and control optimization was done by a simple optimization scheme. A simpler modeling method, along with a comparison of several redundant 
control schemes was proposed by Cheng[6]. A computationally fast methodology for multi-fingered grasping applications, Dynamics Force Distribution, is presented by Zheng[7]. Junkins et al.[8], propose a novel methodology for coordination of redundant sets of actuators in multi-body systems with a weighted pseudo-inverse, with weights determined by the current work space coordinates

\section{Redundant Actuation in Aircraft}

In aircraft control problems, with the on-set of non-traditional control surfaces, the redundant actuation methods have taken a center stage, in recent times, with increase in computation power and hardware capacities. Prioritization of redundant actuator sets is one of the methods of handling redundancy in aircraft control, such schemes, called "Daisy Chain Control Allocation" were first demonstrated by Buffington[9]. When the redundant sets of actuators are actually constrained by their operation limits, Durham[10] proposes constrained control allocation schemes. Bodson[11] presents an overview of constrained control allocation strategies. In the presence of actuator constraints, the pseudo-inverse solution cannot meet the demanded accelerations computed from a square plant. In the light of the same, direct allocation, which Bodson poses as a linear programming problem, presents a highly desirable candidate for real-time application. Singla[12] proposes an approximation approach to recursively allocate using adaptive distribution functions addressing the high dimensionality of the control space with computational efficiency with an application to the morphing problem.

\section{Spacecraft Applications}

In spacecraft attitude control problems, the Control Moment Gyros (CMG) and Reaction Wheels form a major class of actuators. Four Single Gimbal CMGs (SGCMGs) 
in a pyramidal configuration, form a redundant set of actuators in the reorientation maneuvers of spacecraft (This might seem inconsistent at the first blush, but redundancy holds due to the fact that the controllability definitions can be extended to the special case of second order mechanical systems, as has been done by Hughes and Skelton [13]. We shall discuss the redundant actuation with emphasis on the second order mechanical systems in particular and will have to modify the definition of redundant actuation, as external forces cannot influence the kinematics directly but are coupled in a cascade form of differential equations). Control laws can only be implementable, if consistent with actuator dynamics. In some cases, the actuator dynamical models have been included in the control law design. Design of a steering law for redundant SGCMGs was modified by Bedrossian [14] to avoid such singular configurations of the CMGs. Schaub, et al.[15] extended this methodology to obtain control laws for variable speed CMGs, essentially, they introduced additional redundancy to allow a better redistribution of the internal momentum.

Having looked at some applications of redundantly actuated dynamical systems, let us point out some distinctive features of systems with redundant actuation.

\section{Redundantly Actuated Dynamical Systems}

Dynamical systems with redundant actuators are found to possess some distinctive features. The controllability of these systems is, of course, much higher than the ordinary, underactuated dynamical systems. We demonstrate the same in what follows and mention certain other things that a designer may wish to consider with regard to utilizing the actuator redundancy. We shall expand upon those topics in later chapters. 


\section{Controllability}

As is obvious from the name, we should expect redundantly actuated dynamical systems have improved controllability. In the state space model of a linear system,

$$
\dot{x}=A x+B u
$$

$A \in \Re^{n \times n}, B \in \Re^{n \times m}, x \in \Re^{n}, u \in \Re^{m}$, the controllability grammian is given by

$$
\mathcal{C}\left(t_{0}, t_{f}\right)=\int_{t_{0}}^{t_{f}} \Phi\left(t_{0}, \tau\right) B(\tau) B(\tau)^{T} \Phi\left(t_{0}, \tau\right)^{T} d \tau
$$

where $\Phi\left(t_{0}, \tau\right)$ is the state transition matrix and $B$, the control influence matrix (also called the distribution matrix). Assuming $B$ has a full row rank, which usually is a fair assumption owing to the fact that each actuator which is installed and operated independently affects the states of the system differently, we have a mapping between the space of controls and the state space that is atypical to the conventional dynamical systems. While the conventional mapping frequently is from a lower dimensional control space to a higher dimensional state space (under actuated case), this class of redundant systems under discussion maps inputs from a higher dimensional space of controls to a lower dimensional state space $m \geq n$. We already know that such systems are endowed with special properties. One such property is what we call super controllability, explained below.

A careful look at the controllability grammian reveals the fact that the grammian is positive definite at every interval $\left(t_{0}, t_{f}\right)$ of time over which $\Phi\left(t_{0}, t\right)$ is of rank $n$ at (atleast one instant) $t \in\left(t_{0}, t_{f}\right)$. This is due to the full rank structure of the control distribution matrix $B$. Therefore, for $t_{f}=t_{0}+\Delta t, \Delta t \rightarrow 0$, we have the controllability grammian $\mathcal{C}\left(t_{0}, t_{0}+\Delta t\right)>0$, owing to the fact that $B$ has full row rank. This implies that we can, in principle, travel from any point to any other 
point in the state space, indeed a nice property. This property also carries forward for second order mechanical systems owing to the extension of the definitions given by Hughes and Skelton[13]. We are hence motivated to investigate the robustness properties of this class of systems.

\section{Other Features}

Now, Robustness is an important issue in these systems, for, most heavily redundant systems (the systems we are considering for distributed control applications), are poorly modeled. Thus the control methodology or policy should be robust to parameter variations of the plant model. Thus we must face the dual curses of high dimensionality and model uncertainty.

Another issue in the so-called, hyper-redundant actuation strategies is that the redundancy frequently needs to have some kind of system specific coordination. These issues are addressed to some extent in this thesis.

\section{Organization of the Thesis}

The Eigenstructure assignment (EsA) scheme is introduced in chapter II. The assignable subspace of eigenvectors concept is then introduced. It is then shown that for redundantly actuated dynamical systems, in particular, an orthonormal target set is always exactly assignable. A preferred target choice of close loop is then suggested. EsA scheme is then specialized to second order mechanical systems. A novel 2 step EsA scheme is proposed (partial EsA), for assignment of a target real set of closed loop eigenvectors to a second order system. This procedure is then compared with traditional algorithms and applications of the same are discussed. The eigenstructure assignment scheme is then applied to a redundantly actuated multi-link manipulator 
model and the robustness to plant parameter perturbations is demonstrated.

Chapter III discusses coordination among redundant actuator sets. A trained weighted pseudoinverse approach to redundant control via state feedback is presented. This methodology is then demonstrated on the multilink example of chapter II. The EsA approach is then applied to handle Hyper-redundancy in actuation. Distributed Control problems, where there are arrays of redundant actuators to control structures are proposed. A solution of these problems via EsA approach is presented by an adaptive aggregation approach. These details are discussed in chapter IV. In chapter II, we first introduce the EsA scheme. 


\section{CHAPTER II}

\section{EIGENSTRUCTURE ASSIGNMENT}

The Eigenstructure assignment methodology for robust multivariable feedback is understood and considered to be a useful tool in control of dynamical systems via state and output feedback[16]. After the pole placement strategy was extended to multivariable systems by Wonham [17], it was soon recognized that the additional freedom in the feedback gain matrix could be utilized to assign some components of eigenvectors. Srinathkumar [18], first proposed the idea of assignability of some components of the closed loop eigenvectors by choosing the gain matrix of the state and output [19]. Moore [20] derived necessary and sufficient conditions for this procedure. Sinswat, et al.[21] established the assignable subspace of eigenvectors that could be assigned. Numerical methods for well conditioned solutions were later arrived at by Kautsky, et al. [22]. Bhattacharya, et al. [23, 24] solved the problem of eigenstructure assignment by a solution of the a matrix equation, the Matrix Sylvester Equation.

In classical problems, the case with lesser number of actuators are normally considered. Therefore it is impossible to assign exactly a given target set of eigenvectors. Rew, Junkins and Juang [25] present a multi-objective optimization criteria to minimize the error between the assigned eigenvectors and the target set. The application of this multivariable feedback control scheme to flexible spacecraft by an extension considering second order state space representations of mechanical systems was pioneered by Juang [26, 27]. Later, Kim, et al.[28], specialized the Eigenstructure assignment procedure to the second order mechanical systems by establishing the second order Sylvester Equation procedure. Further details on these different schemes and algorithms for eigenstructure assignment are also summarized in Junkins and Kim 
$[29]$.

The flexibility of the methodology to allow the designer to explore the arbitrariness of the closed loop admissible eigenvectors, in addition to the pole assignment makes the method a popular tool for feedback gain calculations. It is well known that near orthogonality of the closed loop eigenvectors is desirable to minimize sensitivity of the eigenvalue placement to the model errors. Arbitrary selection of the closed loop eigenvectors can lead to a highly sensitive gain matrix calculation. Hence the generation of a well conditioned closed loop eigenvector matrix is a key issue to achieve robustness to plant parametric uncertainties. Let us now consider the basics of this design process.

\section{A. Eigenstructure Assignment Methodology}

Consider the linear dynamical system in the state space form.

$$
\dot{x}=A x+B u
$$

with system matrix, $A \in \Re^{n \times n}$, control influence matrix, $B \in \Re^{n \times m}$ and a state feedback control law, $u=G x$. This would give us a closed loop system,

$$
\dot{x}=(A+B G) x
$$

with corresponding closed loop eigenvalue problems written as

$$
\begin{aligned}
(A+B G) \phi_{i} & =\lambda_{i} \phi_{i} \\
(A+B G)^{T} \psi_{i} & =\lambda_{i} \psi_{i}
\end{aligned}
$$

where the left and the right eigenvector sets are normalized according to the relations $\phi_{i} \phi_{i}^{T}=1, \psi_{i}^{T} \phi_{j}=\delta_{i j}$. This implies that, we can, in principle dictate the closed loop 
properties by tailoring the gain matrix, $G$. Let the parameter vector, $h_{i}$ be defined by the relation, $h_{i}=-G \phi_{i}$. Now, the right eigenvalue problem can be rewritten as,

$$
\left(A-\lambda_{i} I\right) \phi_{i}=B h_{i}
$$

when written in matrix form, this equation becomes

$$
A \Phi-\Phi \Lambda=B H
$$

where $\Phi=\left[\phi_{1}, \phi_{2}, \ldots, \phi_{n}\right], \Lambda=\operatorname{diag}\left[\lambda_{1}, \lambda_{2}, \ldots, \lambda_{n}\right]$ and $H=\left[h_{1}, h_{2}, \ldots, h_{n}\right]$. This is a matrix equation belonging to the class of generalized Lyapunov Equations called the Sylvester Equation[23, 24]. The solution of this equation gives us the eigenvector set achieved by a given state feedback gain matrix.

\section{Robustness}

The robustness of this scheme is derived from a theorem due to Patel and Toda, [30]. We state the theorem and discuss the robustness properties.

Theorem II.1 (Patel and Toda [30]) Consider the asymptotically stable closed loop system $\dot{x}=A_{C L} x$. The perturbed closed loop system matrix, $A_{C L}+\Delta$ of the original systems remains asymptotically stable for all perturbations, $\Delta$, bounded by

$$
\|\Delta\|<\frac{\min \left[-\Re \lambda\left(A_{C L}\right)\right]}{\kappa\left(\Phi_{C L}\right)}
$$

where, $\Phi_{C L}$ is the closed loop modal matrix, $\kappa($.$) is the conditioned number defined$ as the ratio of the maximum to the minimum singular values of the matrix, (.) and $\Re\{$.$\} , denoting the real part of \{$.

Proof Original proof given by Patel and Toda [30] uses a transient response formation. An elegant proof of this theorem using simple matrix norm results is given 
by Kautsky et al.[22]. A constructive proof using Lyapunov Theory is presented by Junkins and Kim [29]. Its relationship with the stronger Bauer Fike Theorem [31] is discussed by Junkins and Kim[32].

Hence by choosing the closed loop modal matrix, $\Phi_{C L}$, to be well conditioned, $\kappa(\Phi)=$ 1, whenever possible, we could in principle, bound the unstructured perturbation matrix, by a judicious choice of the minimum eigenvalue and thus design the feedback gain matrix to achieve robustness to the perturbation.

\section{Assignability of Eigenvectors}

Perfect conditioning of the closed loop modal matrix, in general is not possible. When the systems are under actuated, there only some components of the closed loop eigenvectors that can be assigned arbitrarily by the designer. This was pointed out by Srinathkumar[18] and his theorem is given in the appendix. We present a slightly different argument revealing a nice decomposition of the assignable space, along with its dimensionality.

Consider the closed loop system of the underactuated linear system, 2.2, i.e., $n<$ $m$, with the feedback gains computed from, $K=\left(B^{T} B\right)^{-1} B^{T}\left[A-\Phi_{T} \Lambda_{T} \Phi_{T}^{T}\right]$. Substituting this feedback control gain, we get the following closed loop eigenvalue problem.

$$
\begin{array}{r}
(A+B K) \Phi_{T}=\Phi_{T} \Lambda_{T} \\
\left(I-B\left(B^{T} B\right)^{-1} B^{T}\right) A \Phi_{C L}+B\left(B^{T} B\right)^{-1} B^{T} \Phi_{T} \Lambda_{T} \Phi_{T}^{-1} \Phi_{C L}=\Phi_{C L} \Lambda_{C L}
\end{array}
$$

where, $\Phi_{C L}$ is the closed loop eigenvector set achieved by the gain matrix. We can clearly notice the orthogonal projector, $B\left(B^{T} B\right)^{-1} B^{T}$ and its orthogonal complement. It is known that this projector projects on to the range space of $B$. If $Q_{R}$ of order $n \times m$, (assuming a row rank of $m$ for $B$ ), is a matrix with its columns forming 
an orthonormal set spanning the range of $B$,(This can always be determined from the SVD of the projector $[33,34])$ the projector can be written as $B\left(B^{T} B\right)^{-1} B^{T}=$ $Q_{R} Q_{R}^{T}$. Its orthogonal complement being given by another orthonormal set $Q_{N}$, $\left(I-B\left(B^{T} B\right)^{-1} B^{T}\right)=Q_{N} Q_{N}^{T}$. Thus the closed loop eigenvalue problem becomes,

$$
Q_{N} Q_{N}^{T} A \Phi_{C L}+Q_{R} Q_{R}^{T} \Phi_{T} \Lambda_{T} \Phi_{T}^{-1} \Phi_{C L}=\Phi_{C L} \Lambda_{C L}
$$

Note the clear separation of the closed loop eigenstructure. From this it is clear that the least squares solution of the gain matrix has acted as an orthogonal projector, only assigning $m$ components of each mode $\left(Q_{R}\right.$, being the orthonormal basis for the range space of $\mathrm{B}$ ). The null space of the projector clearly retains the open loop eigenstructure. This agrees with Srinathkumar[18],and is an important revelation, as we have found an associated orthonormal set, $Q_{R}$. We found that this is one choice orthogonal target set of eigenvectors to minimize the feedback gain matrix, by trial and error computation, as will be pointed out later.

B. Robust Eigenstructure Assignment (EsA) for Redundantly Actuated Systems

Systems with greater number of control inputs than the states in the plant model $m \geq n$ are said to be redundantly actuated. We find that we can say much more about the feedback gains in the case of the redundantly actuated systems. We also note that the results of this section also hold for the case of square plants (Plants with actuators equal to the number of states). Thus we have the following (obvious) lemma for redundantly actuated state feedback solutions.

Lemma II.1 For a redundantly actuated linear dynamical system,2.1, a target orthonormal set of eigenvectors is exactly assignable, for a given target set of distinct closed loop eigenvalues, $\Lambda_{C L}$. 
Proof We need to show that, for every desired orthonormal eigenvector set, $\Phi$, of the closed loop, there exists a feedback gain matrix, $G$, which can place the poles at the target location, $\Lambda_{C L}$.

Given, an orthonormal target modal matrix for the closed loop, we can always compute the right hand side of the matrix Sylvester equation,

$$
A \Phi-\Phi \Lambda_{C L}=-B G \Phi \equiv B H
$$

The feedback gain matrix is then obtained by taking the minimum norm solution of the resulting redundant equation sets and is given by,

$$
H_{\text {min } n o r m}=B^{\dagger}\left(A \Phi-\Phi \Lambda_{C L}\right)
$$

where $B^{\dagger} \equiv W B^{T}\left(B W B^{T}\right)^{-1}$. Note that the exact solution is possible only due to the assumed full rank structure of $B$ and the dimension of its range space. In the standard underactuated case, this attainment of an arbitrary set of eigenvectors is impossible, in general and we are better off with minimizing some criterion using the approach of Rew, et al.[25]. Substituting the calculated gain, and forming the closed loop system, we get,

$$
\dot{x}=(A-B G) x=\left(A-B B^{\dagger}\left(A \Phi-\Phi \Lambda_{C L}\right)\right) x=\Phi \Lambda_{C L} \Phi^{T} x .
$$

Thus the closed loop modal matrix is assigned exactly.

We are now ready to state an evident fact and a useful consequence of this lemma.

Theorem II.2 A redundantly actuated linear dynamical system is asymptotically stable for all additive perturbations of the plant parameters, bounded by, $\|\Delta\|<$ $\min \left[-\Re\left\{\lambda\left(A_{C L}\right)\right\}\right]$, where, $A_{C L}$, is the closed loop system 
Proof Using the Patel and Toda theorem II.1, the bound on the allowable plant parameter perturbations for the system, 2.1 , to be asymptotically stable is that

$$
\|\Delta\|<\frac{\min \left[-\Re\left\{\lambda_{i}\left(A_{C L}\right)\right\}\right]}{\kappa(\Phi)}
$$

Clearly, when, the linear system is redundantly actuated, from lemma, II.1, a given orthonormal target closed loop modal matrix is always exactly assignable. Therefore, if $\Phi_{C L}$, is the target modal matrix, and if it were chosen orthonormal (or unitary), $\kappa(\Phi)=1$. This proves the theorem.

We can easily see the additional freedom the designer has. For example, we can place the smallest of the closed loop poles at a chosen location, away from the imaginary axis in the open left half plane, to bound the parametric uncertainty possible and still guarantee asymptotic stability of the system.

Example: The following is an example system with 2 states and 3 control inputs, where we have assigned the closed loop eigenvalues and eigenvectors at a target set to demonstrate the aforementioned properties of the redundantly actuated dynamical systems.

$$
\begin{array}{r}
A=\left[\begin{array}{ll}
10 & 2 \\
5 & 20
\end{array}\right], B=\left[\begin{array}{ccc}
2 & -1 & 1 \\
3 & 2 & -1
\end{array}\right] ; \\
\Phi_{\text {target }}=\left(\begin{array}{cc}
\cos \theta & \sin \theta \\
-\sin \theta & \cos \theta
\end{array}\right), \Lambda_{C L \text { target }}=\left(\begin{array}{cc}
-10 & 0 \\
0 & -15
\end{array}\right)
\end{array}
$$

$\Phi_{\text {target }}$ is the target eigenvector set generated by varying the parameter $\theta$ from $0^{0}$ to $45^{0}$. We then calculate the gain matrix for the state feedback from the matrix Sylvester equation. The resulting closed loop system properties are analyzed as shown in figure 1. In the first subplot, the eigen value assignment error $e \equiv \Lambda_{\text {target }}-\Lambda_{C L}$, is 
plotted as a function of the parameter $\theta$, used to generate the target eigenvector set. Second subplot shows the magnitude of the feedback gain matrix as a function of the parameter. The third subplot shows the condition number of the closed loop modal matrix showing unity for all targets. This shows the exact assignment of the target modal matrix by the scheme.
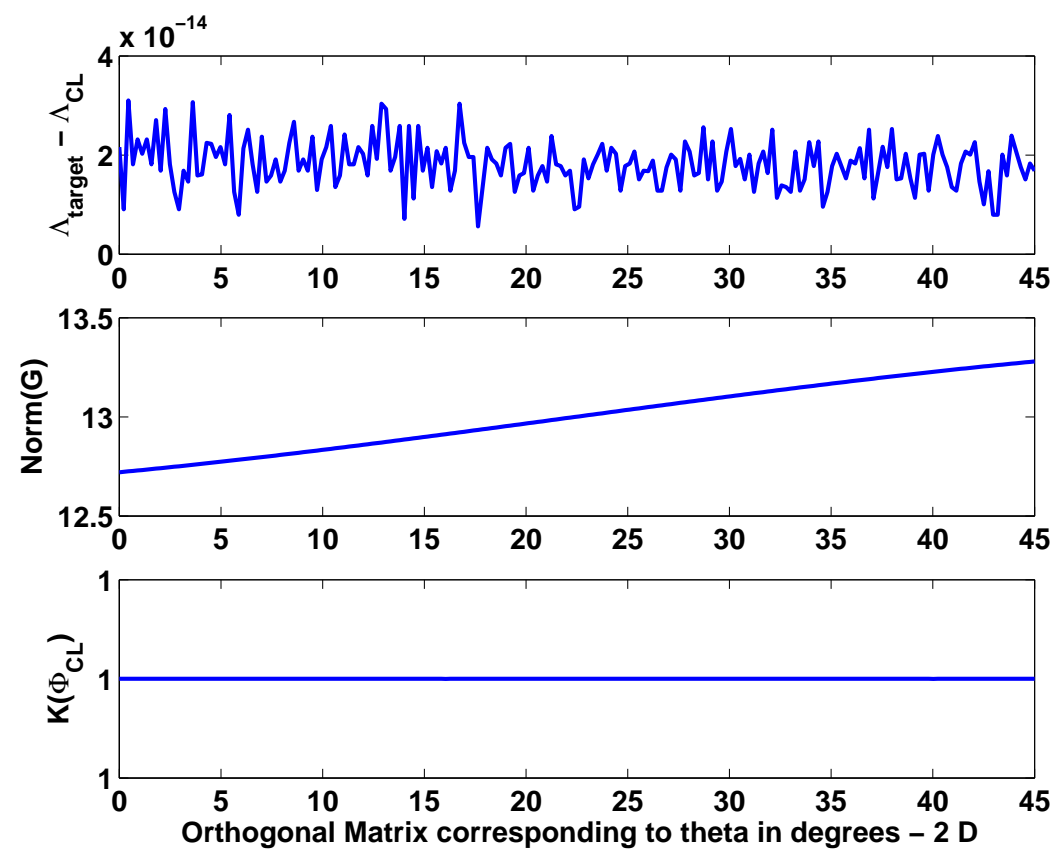

Fig. 1. Eigenstructure assignment: 2D example

\section{Target Sets of Eigenvectors}

The varying feedback gain matrix magnitude in the figure 1 , indicates that there exist a target orthonormal set which could minimize the magnitude of the state feedback gain. This motivates us to pose the following optimization problem. We want to find an symmetric matrix that is closest to a given matrix. This is very much similar to the famous Procrustes problem in psychometrics[34]. We would like to find 
an orthogonal modal matrix to minimize the cost function,

$$
\min _{\Phi}\|B G\|_{F}=\min _{\Phi}\left\|A-\Phi \Lambda \Phi^{T}\right\|_{F}
$$

One particular choice inspired from Juang and Maghami [27] has the property of staying as close as possible to the open loop modal matrix, given by, $\Phi_{\text {target }}=U_{E} V_{E}^{H}$, where, $A=E \Lambda_{O L} E^{-1}$ is the spectral decomposition of the open loop system matrix, and $E=U_{E} \Sigma_{E} V_{E}$, is the singular value decomposition of $E$. The performance index, with this choice of the target eigen vector set becomes,

$$
\|B G\|=\operatorname{tr}\left(A^{T} A\right)+\operatorname{tr}\left(\Lambda^{2}\right)-2 \operatorname{tr}\left(V_{E}^{H} \Lambda V_{E} \Sigma_{E} V_{E}^{H} \Lambda_{O p e n l o o p} V_{E} \Sigma_{E}^{-1}\right)
$$

We are interested in real time computation of the gain minimizing target modal matrix. The parametrization of $\Phi$ to make the determination of a minimizing target, an optimization problem is therefore avoided.

Observations: In contrast to the suggestions of Juang and Maghami [27], it was observed that, a better target for closed loop modal matrix is the unitary matrix spanning the range space of $B$. With this set as the target set of closed loop eigenvectors, the feedback gains were observed considerably smaller in magnitude in comparison with the gain magnitudes when open loop system range was used as an orthonormal matrix. A random test case is shown in figure 2. This is not surprising considering the result in equation 2.10, and the fact that the range of B determines the closed loop modal matrix. But, more concrete results or a general proof could not be obtained at present time. Also, substituting, the product $A B$ would do apparently do no better in the achievement of lower gains as this also belongs to the same Krylov subspace [34] (or the controllability space), in terms of the linear algebraic systems. 


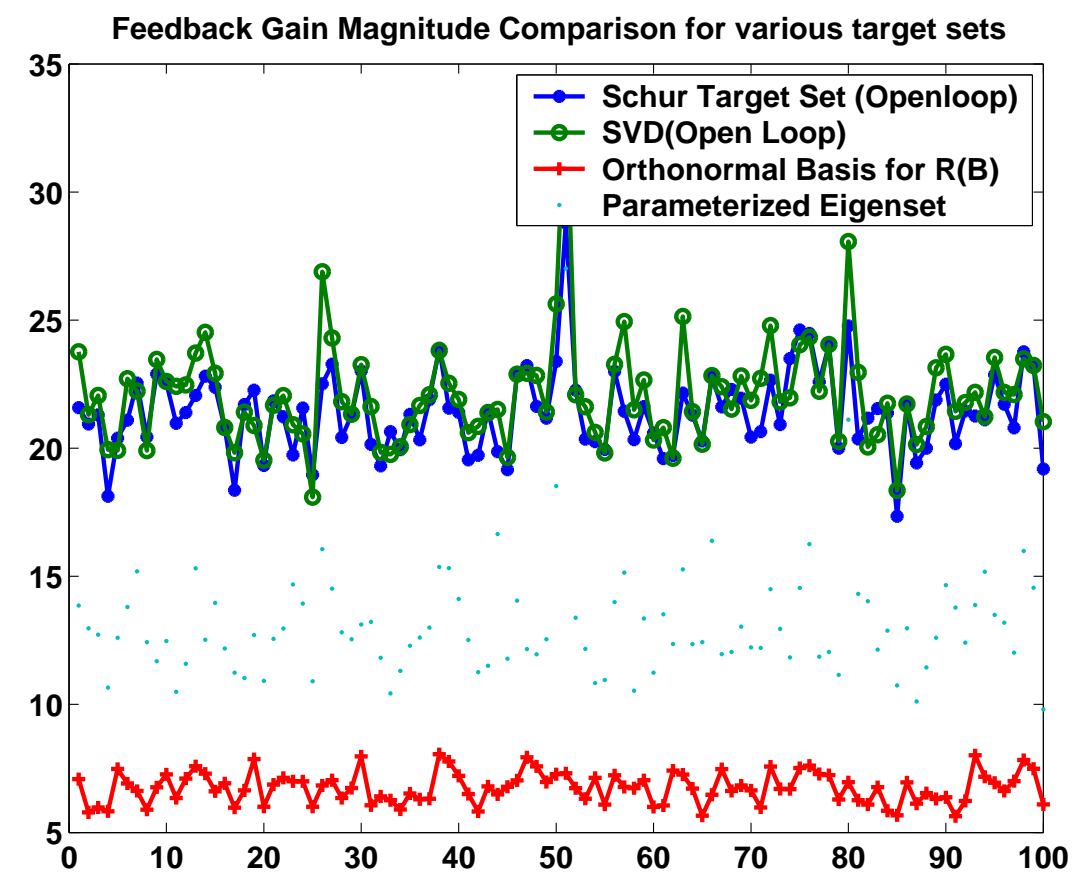

Fig. 2. Comparison of various orthonormal target sets for feedback gain minimization

C. Second Order Mechanical Systems: Partial Eigenstructure Assignment

Extension of the Eigenstructure Assignment scheme to second order systems has been discussed extensively in the literature. Among other methods, the sequential eigenstructure assignment by Juang [27] and the second-order Sylvester Equation approach by Kim, et al.[28] are particularly attractive.

When we have the same number of actuators as the states of the system, it was found that there exists yet another attractive and simple methodology for gain computation for simultaneous feedback of position and velocity and partial eigenstructure assignment. The following section summarizes the same. 


\section{Partial Eigenstructure Assignment of Second Order Systems}

Most of the second order mechanical systems, in practice allow the assignability of a part of the eigenstructure. This is because, the external forces do not apply directly on the kinematics part of the dynamical system. It therefore follows from the developments of Srinathkumar[18], that only $n$ components (of the total $2 n$ state variables, of course assuming there are atleast $n$ ) of each mode can be assigned arbitrarily. This is sufficient for most practical applications, if we desire a complex conjugate pair of target set of closed loop eigenvalues. This is because of the following structure of the modal matrix for any linear second order mechanical system.

$$
\Theta=\left(\begin{array}{cc}
\Phi & \bar{\Phi} \\
\Phi \Lambda & \overline{\Phi \Lambda}
\end{array}\right)
$$

Clearly, because of the constraints from the kinematics and the complex conjugate eigenvalues of the second order system, the determination of the $n \times n$ principal sub modal matrix, $\Phi$, determines the entire modal matrix. We utilize this fact and try and make the principal sub modal matrix, $\Phi$, as well conditioned as possible. Now we develop a systematic method to do the partial EsA.

Let the second order mechanical system be given by

$$
M \ddot{x}+C \dot{x}+K x=B u
$$

with $M \in \Re^{n \times n}, x \in \Re^{n}, u \in \Re^{m}, B \in \Re^{m \times n}$, and $m \geq n, \operatorname{rank}(B)=n$. With a position and velocity feedback, $u=G x=G_{1} x+G_{2} \dot{x}$, the closed loop eigenvalue problem for the second order mechanical system is given by

$$
\left(M \lambda_{i}^{2}+\left(C-B G_{2}\right) \lambda_{i}+\left(K-B G_{1}\right)\right) \phi_{i}=0
$$


where, $\lambda_{i} \in \mathcal{C}^{n}=\lambda_{R i}+j \lambda_{I i}, \lambda_{R i}, \lambda_{I i} \in \Re$ and $j^{2}=-1$. We assign a set of distinct complex target poles and their conjugates are assigned automatically. Let us assume for simplicity that the $n \times n$ principal mode matrix is real. The analysis can be repeated with out loss of generality to derive the matrix equation in the complex target as well, without loss of generality. Separating the real and imaginary parts of the above equation, we get the following two conditions,

$$
\begin{aligned}
\left(M\left(\lambda_{R i}^{2}-\lambda_{I i}^{2}\right)+\left(C-B G_{2}\right) \lambda_{R i}+\left(K-B G_{1}\right)\right) \phi_{i} & =0 \\
j\left(2 M \lambda_{R i} \lambda_{I i}+\left(C-B G_{2}\right) \lambda_{I i}\right) \phi_{i} & =0
\end{aligned}
$$

Clearly the equations above represent a cascade and one of them is the matrix Sylvester equation, we saw earlier. From the above conditions, together with the fact that we can assign a target $n \times n$ principal modal sub matrix exactly owing to the fact that the plant is atleast square, we have the following feedback gains.

$$
\begin{array}{r}
G_{2}=B^{-1}\left(C+2 M \Phi \Lambda_{R} \Phi^{T}\right) \\
G_{1}=B^{-1}\left(M \Phi\left(\Lambda_{R}^{2}-\Lambda_{I}^{2}\right) \Phi^{T}-2 M \Phi \Lambda_{R}^{2} \Phi^{T}+K\right)
\end{array}
$$

It is found that these gains assign the closed loop poles along with the principal target modal sub-matrix exactly (subject to appropriate scaling and complex magnitude considerations). We demonstrate these features with the following example problem (example 5.4 from Junkins and Kim [29]) and discuss the positive features of the algorithm proposed above. 


\section{Example}

Consider the undamped open loop second order system with the following parameters,

$$
M=\left(\begin{array}{lll}
1 & 0 & 0 \\
0 & 1 & 0 \\
0 & 0 & 1
\end{array}\right), K=\left(\begin{array}{ccc}
20 & -10 & 0 \\
-10 & 30 & -20 \\
0 & -20 & 20
\end{array}\right)
$$

With these parameter matrices, consider the target eigenvalues given by, $\Lambda_{\text {target }}=$ $\operatorname{diag}(-0.72+1.24 i,-0.72-1.24 i,-2.23+3.87 i,-2.23-3.87 i,-3.4+5.9 i,-3.4-5.9 i)$, that is $\omega_{1}=1.44, \omega_{2}=4.47, \omega_{3}=6.92, \zeta_{1}=\zeta_{2}=\zeta_{3}=0.5$. The assigned eigenvalues computed from the above methodology and the corresponding closed loop eigenvectors are shown below.

$$
\Lambda=\operatorname{diag}(-0.72+1.24 i,-0.72-1.2 i,-2.2+3.8 i,-2.2-3.8 i,-3.46+5.9 i,-3.46-5.9 i),
$$

$$
\Phi=\left(\begin{array}{ccc}
-0.52218-0.533 i & 0 & 0 \\
0 & -0.11656-0.1935 i & 0 \\
0 & 0 & -0.0737-0.125 i \\
1.0405-0.0267 i & 0 & 0 \\
0 & 1.012-0.022 i & 0 \\
0 & 0 & 1.0051-0.00918 i
\end{array}\right)
$$

The above equations are the representative modes of closed loop system. The other three modes are the complex conjugates of the above modes. At first blush, it may seem that this is not the eigenstructure we wanted. That is due to the bi-orthogonality conditions we imposed for normalization. Upon dividing each column so as to make the principal sub-modal matrix unity, we unravel the following scaled modes (that is, 
divide ith eigenvector with its $i, i$ th entry, in the above set).

$$
\Phi_{\text {scaled }}=\left(\begin{array}{ccc}
1 & 0 & 0 \\
0 & 1 & 0 \\
0 & 0 & 1 \\
-0.72+1.2471 i & 0 & 0 \\
0 & -2.235+3.8711 i & 0 \\
0 & 0 & -3.46+5.9929 i
\end{array}\right)
$$

Clearly, this is the target eigenstructure. We also recover the condition number by this scaling to have the same conditioning as the target modal matrix.

This can be compared with the solution in Junkins and Kim [29] in table 5.3. Using a unitary matrix as a target, the norm of the feedback gains required was $\|G\|_{F}=50.91$, whereas in this case, the norm of the gain matrix was found to be $\|G\|_{F}=37.363$.

Thus we can see from the above that we need not always use a projection method to stay as close as possible to a known, well conditioned system. It is also of consequence to note: If we concentrate on the assignable sub modal matrix, noting that the second order mechanical systems have a special structure and with a square plant, then we can always assign exactly the assignable target sub matrix.

\section{Features of the Partial EsA Algorithm}

Whenever possible, the partial EsA algorithm has the following advantages and disadvantages when compared to its cousins proposed in Juang and Maghami [27], Juang et al.[26] and Kim, et al.[28].

- The algorithm above is computationally less expensive than its cousins. Juang 
[26] algorithm uses the SVD for each eigenvalue/vector assignment and a matrix inverse is required, whereas we need only one matrix inverse. Juang's method[27] requires a QR step and an SVD for each eigenvalue/vector as the procedure uses angle between subspaces. Kim's approach is actually quite similar to the present approach, but does not use the same matrix manipulations as the above algorithm.

- This algorithm doesn't need the modal damping assumption which is important for Kim's algorithm. The damping can be general and even asymmetric. In fact, the only restriction we need in the above development is to have an invertible (or full rank) control influence matrix and a positive definite mass matrix.

- The drawback of this method is that we need at least $n$ actuators for $2 n$ state variables in the second order form. This can be relaxed but we cannot assign the target submodal matrix. The method needs further development for those cases (May be using some kind of a projection criterion).

- The method needs an extension to assign complex principal modal sub matrices. This brings about a change in the structure of the conditions that need to be satisfied.

- An arbitrary selection of target eigenvector set can lead to very high gains and a sensitive feedback controller. Therefore we need to select the target modal matrices judiciously. 


\section{CHAPTER III}

\section{EXAMPLE APPLICATION}

In this section we shall demonstrate the utility of the EsA methodology, by controlling a redundantly actuated robotic manipulator. The schematic of the manipulator is shown in figure 3. Assuming unit lengths for the links, the constrained equations

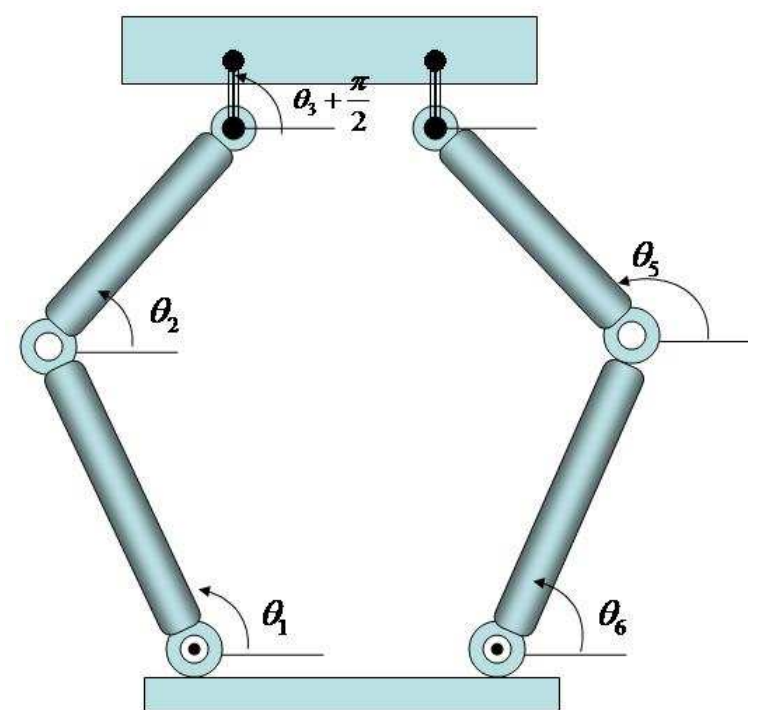

Fig. 3. Schematic of a redundantly actuated robot

of motion of the manipulator are given by

$$
\begin{gathered}
M \ddot{q}+\tilde{G}=\tilde{B} u \\
\tilde{G}=G-F_{1} \\
\tilde{B}=B+F_{2}
\end{gathered}
$$


where $q=\left[q_{L}, q_{R}, q_{P}\right]^{T}=\left[\theta_{1}, \theta_{2}, \theta_{6}, \theta_{5}, \theta_{3}, x_{c 3}, y_{c 3}\right]^{T}$ are the unconstrained degrees of freedom of the left, right arms and the platform respectively. The control input $u=\left[u_{1}, u_{2}, u_{6}, u_{5}, u_{3}, f_{x}, f_{y}\right]^{T}$ are the joint torques $u_{i}$ at the $i t h$ joint and the resultant forces $f_{x, y}$ due to thrusters at the payload mass center. The control effectiveness matrix, is given by the principle of virtual work as

$$
B=\left[\begin{array}{ccccccc}
1 & -1 & 0 & 0 & 0 & 0 & 0 \\
0 & 1 & 0 & 0 & -1 & \sin \theta_{23} & -\cos \theta_{23} \\
0 & 0 & 1 & -1 & 0 & 0 & 0 \\
0 & 0 & 0 & 1 & -1 & \sin \theta_{53} & -\cos \theta_{53} \\
0 & -1 & 0 & -1 & 1 & 0 & 0 \\
0 & 0 & 0 & 0 & 0 & 1 & 0 \\
0 & 0 & 0 & 0 & 0 & 0 & 1
\end{array}\right]
$$

where $\theta_{i j}=\theta_{j}-\theta_{i}$ and $F_{1}, F_{2}$ are the forces due to geometric constraints. The geometric constraints are given by

$$
\begin{array}{r}
\cos \theta_{1}+\cos \theta_{2}+\frac{1}{2} \cos \theta_{3}-x_{c 3}=0 \\
\sin \theta_{1}+\sin \theta_{2}+\frac{1}{2} \sin \theta_{3}-y_{c 3}=0 \\
\cos \theta_{6}+\cos \theta_{5}-\frac{1}{2} \cos \theta_{3}-x_{c 3}+D=0 \\
\sin \theta_{6}+\sin \theta_{5}-\frac{1}{2} \sin \theta_{3}-y_{c 3}=0
\end{array}
$$

The constraint jacobian being given by

$$
J=\left(\begin{array}{ccccccc}
-\sin \theta_{1} & -\sin \theta_{2} & 0 & 0 & -\frac{1}{2} \sin \theta_{3} & -1 & 0 \\
\cos \theta_{1} & \cos \theta_{2} & 0 & 0 & \frac{1}{2} \cos \theta_{3} & 0 & -1 \\
0 & 0 & -\sin \theta_{6} & -\sin \theta_{5} & \frac{1}{2} \sin \theta_{3} & -1 & 0 \\
0 & 0 & \cos \theta_{6} & \cos \theta_{5} & -\frac{1}{2} \cos \theta_{3} & 0 & -1
\end{array}\right)
$$


giving the jacobian derivative,

$$
\dot{J}=\left(\begin{array}{ccccccc}
-\dot{\theta}_{1} \cos \theta_{1} & -\dot{\theta}_{2} \cos \theta_{2} & 0 & 0 & -\frac{1}{2} \dot{\theta}_{3} \cos \theta_{3} & 0 & 0 \\
-\dot{\theta}_{1} \sin \theta_{1} & -\dot{\theta}_{2} \sin \theta_{2} & 0 & 0 & -\frac{1}{2} \dot{\theta}_{3} \sin \theta_{3} & 0 & 0 \\
0 & 0 & -\dot{\theta}_{6} \cos \theta_{6} & -\dot{\theta}_{5} \cos \theta_{5} & \frac{1}{2} \dot{\theta}_{3} \cos \theta_{3} & 0 & 0 \\
0 & 0 & -\dot{\theta}_{5} \sin \theta_{5} & -\dot{\theta}_{5} \sin \theta_{5} & \frac{1}{2} \dot{\theta}_{3} \sin \theta_{3} & 0 & 0
\end{array}\right)
$$

giving us the constraint forces as

$$
\begin{array}{r}
F_{1}=J^{T}\left(J M^{-1} J^{T}\right)^{-1}\left(J M^{-1} G-\dot{J} \dot{q}\right) \\
F_{2}=-J^{T}\left(J M^{-1} J^{T}\right)^{-1} J M^{-1} B
\end{array}
$$

Thus we are led to the constraint free form of the equations of the manipulator given by

$$
\begin{aligned}
& M_{L} \ddot{q}_{L}+\tilde{G}_{L}=\tilde{B}_{L} u \\
& M_{R} \ddot{q}_{R}+\tilde{G}_{R}=\tilde{B}_{R} u \\
& M_{P} \ddot{q_{P}}+\tilde{G}_{P}=\tilde{B}_{P} u
\end{aligned}
$$

For payload manipulation, we prescribe a reference maneuver. A smooth, nominal, rest-to-rest maneuver is prescribed given by

$$
\begin{array}{r}
q_{\text {pref }}(t)=f(\tau)\left[q_{\text {pref }}\left(t_{f}\right)-q_{\text {pref }}\left(t_{0}\right)\right]+q_{\text {pref }}\left(t_{0}\right) \\
\dot{q}_{\text {pref }}(t)=\frac{d f(\tau)}{d \tau} \frac{\left(q_{\text {pref }}\left(t_{f}\right)-q_{\text {pref }}\left(t_{0}\right)\right)}{\left(t_{f}-t_{0}\right)} \\
\ddot{q}_{\text {pref }}(t)=\frac{d^{2} f(\tau)}{d \tau^{2}} \frac{\left(q_{\text {pref }}\left(t_{f}\right)-q_{\text {pref }}\left(t_{0}\right)\right)}{\left(t_{f}-t_{0}\right)^{2}}
\end{array}
$$

where, $\tau=\frac{\left(t-t_{0}\right)}{\left(t_{f}-t_{0}\right)}$ and the shape function $f(\tau)=\tau^{3}\left(10-15 \tau+6 \tau^{2}\right)$ is chosen, as this nominal reference is known to simultaneously minimize the translational and 
rotational jerk integrals applied to the payload. Assuming that the position and the velocity of the payload coordinates, $q_{p}, \dot{q}_{p}$ are available for measurement, we propose the feedback control law,

$$
u=W \tilde{B}_{p}\left(\tilde{B}_{p}^{T} W \tilde{B}_{p}\right)^{-1}\left[\tilde{G}_{p}+M_{p} \ddot{q}_{p r e f}+M_{p} K\left(q_{p}-q_{p r e f}\right)+M_{p} C\left(\dot{q}_{p}-\dot{q}_{p r e f}\right)\right]
$$

where, the active stiffness and the damping $K, C$ are designed by the second order eigenstructure assignment methodology discussed in chapter II.

To demonstrate the robustness of the control law, 3.17, we introduce perturbations in the manipulator parameters, model errors and initial condition errors as shown in table I. Clearly, these are large model errors and the objective shall be to stay as close as possible to the reference trajectory. The response of the system is shown in the figures 4,5. From figures 4,5, it is apparent that the structure of the simple feedback scheme is robust for a variety of plant parametric uncertainty types. This would not have been possible if the plant was under-actuated as the number of feedback gains to be designed would be lesser and we should be focusing more on stability of the plant than the robustness. Hence redundancy in actuation has been shown to address other objectives of control of dynamic systems rather than stability alone. In the next section, we explore another such feature of redundantly actuated dynamical systems, coordination. 
Table I. Applied additive parameter perturbations to plant model

\begin{tabular}{|l|c|}
\hline Type of Perturbation & Amount of Perturbation (Percentage) \\
\hline$M_{p}$, Elements of Mass Matrix & 60 \\
\hline$q_{p}\left(t_{0}\right)$, Initial conditions & 20 \\
\hline scaled $\tilde{G}$ Model Error & 60 \\
\hline Error in $\ddot{q}_{r e f}$ model parameters & 40 \\
\hline
\end{tabular}
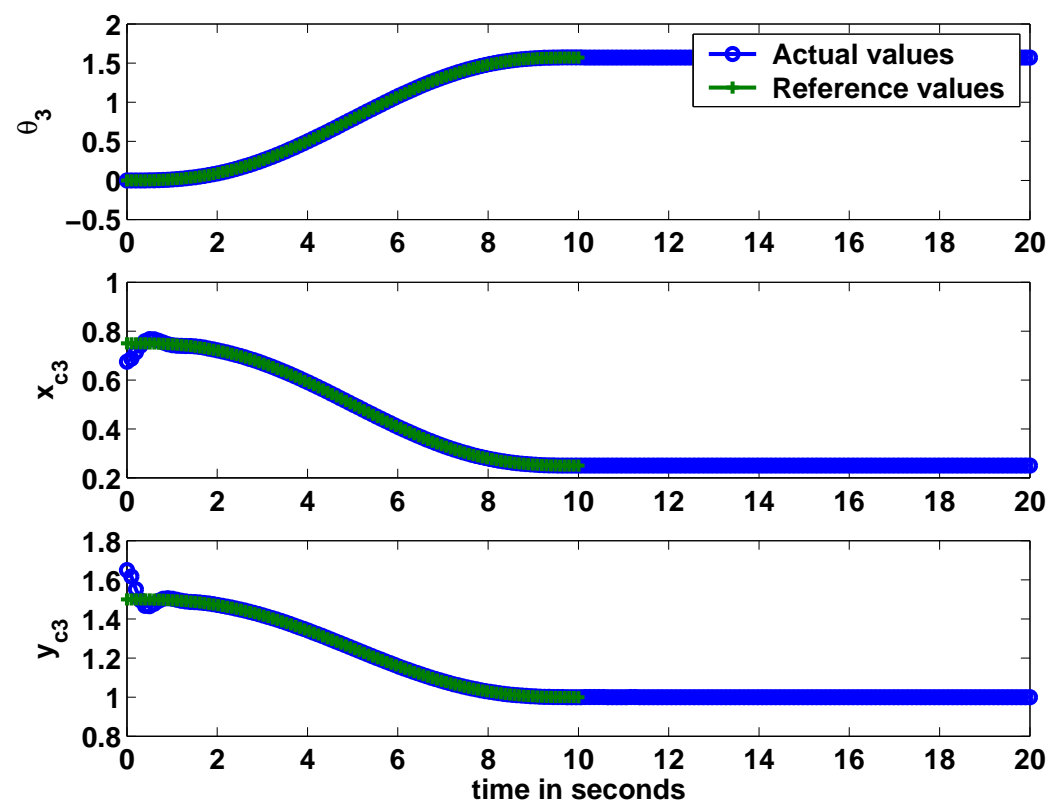

Fig. 4. Robust position tracking of the platform coordinates 

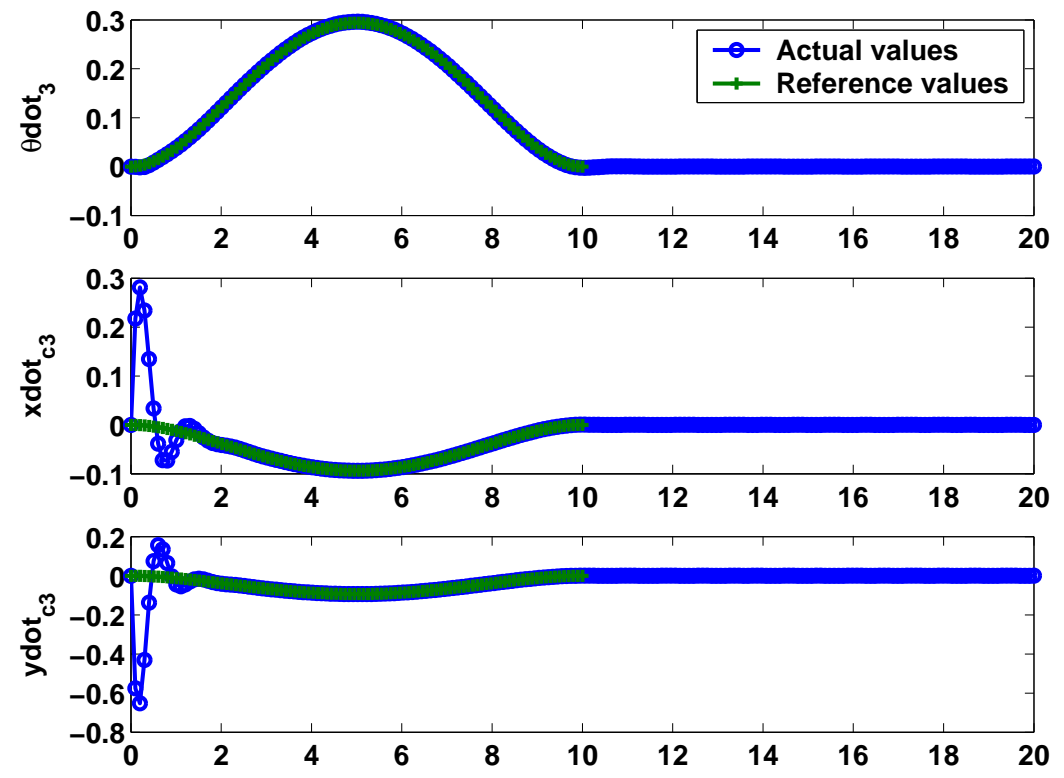

Fig. 5. Robust velocity tracking of the platform coordinates 


\section{CHAPTER IV}

\section{COORDINATION OF REDUNDANT ACTUATOR SETS}

Coordination is an important feature that the redundant actuator sets should possess in order to achieve the global control objective without having conflicting local goals. Junkins and Akella[8] demonstrated coordination among redundant actuators by making the weight matrix a function of one of the state variables. We extend the idea with an objective of improving controllability of the system.

\section{A. Trained Weighting Approach for Coordination}

The central objective of this approach is to determine the influence of each control input towards the state evolution and make the weighting function in the pseudoinverse (3.17), so as to increase the contribution of actuators with maximum effectiveness instantaneously. We achieve this objective by training weight functions, using unit actuator inputs (force/torque). Training involves the propagation of a variety of points in the states space (An evenly distributed grid of points) to derive an effectiveness metric for each actuator in the redundant set, while maintaining zero force input from the rest of the actuators. For example, the distance traveled from each point in the state space in a given time by applying a unit force from only one actuator being a candidate metric measuring the effectiveness of that actuator at that point in the state space. This off-line computation, then gives us data to fit the performance metric as a function of the required variable (some norm in the state space is a good candidate). Thus, we have a knowledge of the relative importance of each actuator in the workspace by repeated training from various feasible points in the given work space. When a dynamical system traverses from one point in the state space to another, the local effectiveness of each actuator in such a transport is being 
approximated grossly by this process.

The idea is illustrated in the figure 6 . Consider the black line to be a part of the operating region. Naturally, at different points of the operating region in the state space, different actuators have different control effectiveness on the system. Given a point, say $x\left(t_{2}\right)$, one such metric to measure effectiveness of actuator would be the distance in the state space, traversed by applying unit actuation force, in one time unit. This when repeated for all actuators gives us a means of comparison between the relative strengths of actuator sets at that given point in the state space. For example, as can be noted from figure 6 , at $x\left(t_{2}\right)$, the most effective actuator would be $u_{2}$, as it takes $x\left(t_{2}\right)$ to $x\left(t_{2}+\Delta\right)$ in $\Delta$ time, which is the largest of the distances by the available controls $u_{1}, u_{2}, u_{3}$. After the positive weight function determination, we could use it in the control implementation. We can extend this procedure from one actuator at a time to two actuators at a time so that we can populate the instantaneous correlation terms in the trained weight matrix. We could use any choice of weight functions for this approximation.

A potential drawback in this scheme is that if a given actuator is highly effective, it will be given the maximum weight and some other actuator with poorer effectiveness may be given only slight torques, leading to some kind of overload on some actuators. The implementation on the redundant robot example (chapter III) showed similar phenomena. It appears from figure 8 that the motor at joint 1 has been given some "rest" as it is ineffective in the region (comparing figures 7 and 8, figure 7 shows control forces different in magnitude from equal weighting approach). This makes complete sense, physically, as in the given maneuver, the payload, being rotated clockwise, requires a greater effort from the elbow and shoulder joints of the right manipulator. 
The left shoulder thus requires only slight effort. The unusually high control effort on each actuator is due to the fact that the system parameters are highly perturbed along with the initial conditions.

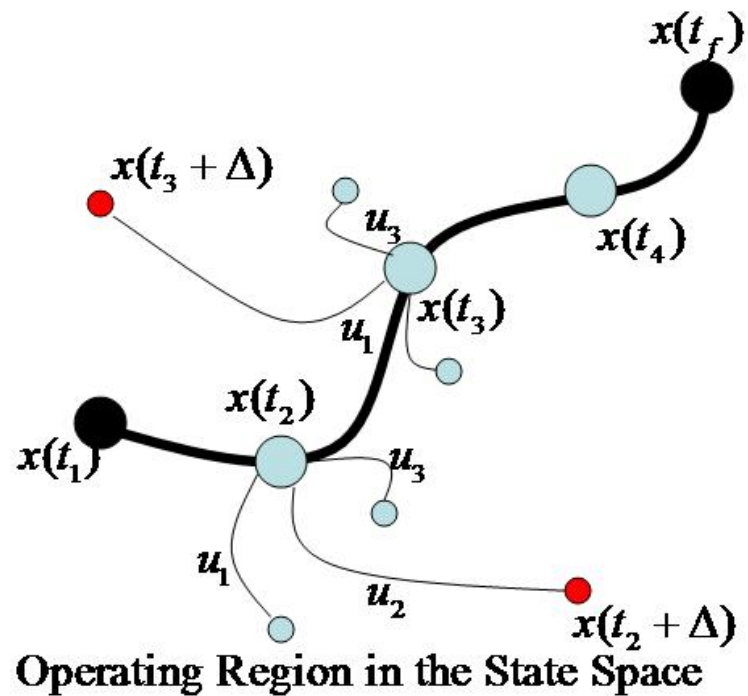

Fig. 6. Schematic illustrating the idea of training the weight matrix approximating effectiveness 


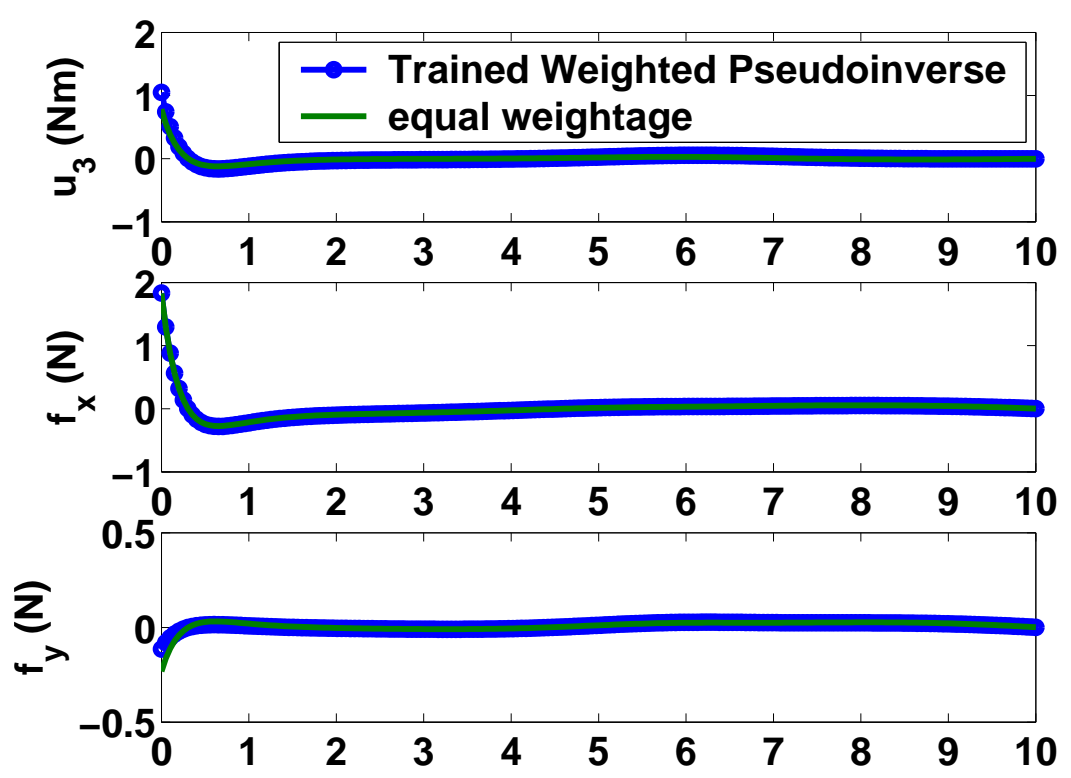

Fig. 7. Control inputs on payload with and without trained weighting
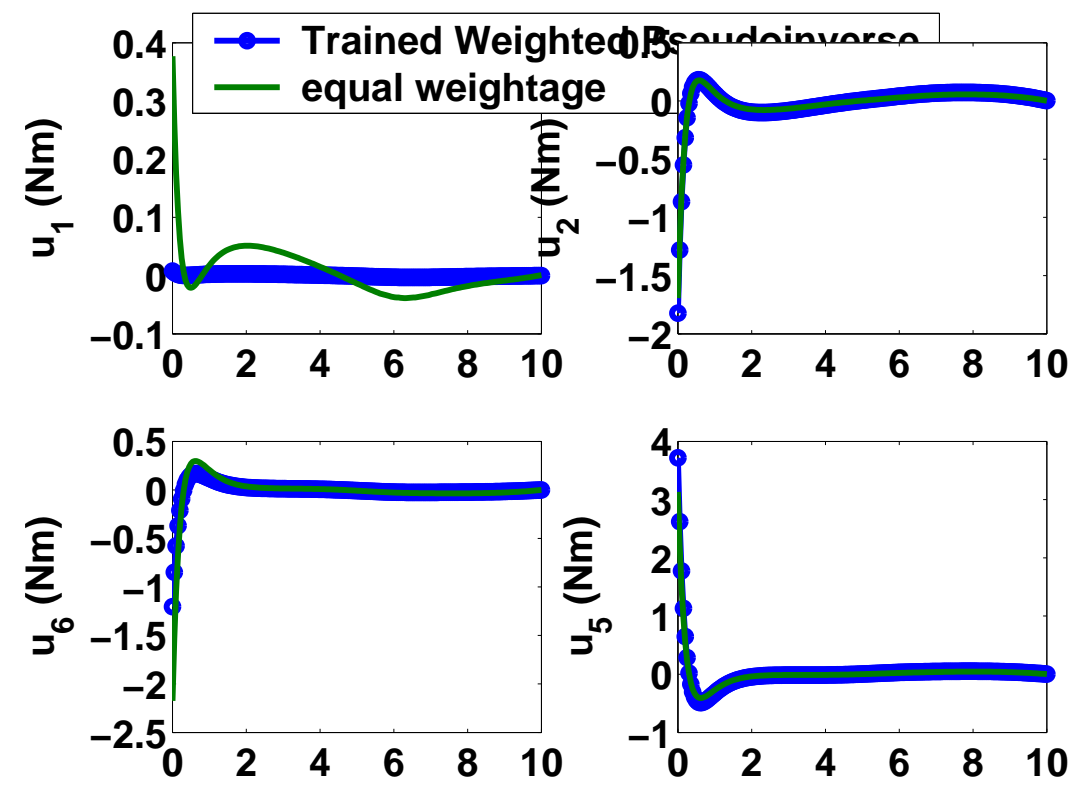

Fig. 8. Control inputs on arm and shoulder joints with and without trained weighting 


\section{CHAPTER V}

\section{APPLICATION TO DISTRIBUTED CONTROL PROBLEMS: HYPER-REDUNDANT ACTUATION}

In smart structures, the actuation is distributed in space. Shape memory alloys and piezo-electric materials are examples for this type of actuators. Nominally, they have inputs at several places and the smart matter delivers a distributed force input to the structures they are installed upon, in accordance with the input voltage signal at that location. It is envisioned that there will be novel smart sensing and actuation technologies using nano-materials in the near future which will increase the input dimensionality by an order of magnitude more than the current smart systems. Such actuators are poorly modeled and the over all system is thereby poorly captured by the physics based models. Hence there is a need for the control synthesis methodology to be insensitive to parametric uncertainties. Stability of such systems which have a continuum of actuators, governed by partial differential equations is a highly developed area. Wang $[35,36]$ pioneered the extension of Lyapunov's direct method to analyze the stability of such systems and is accredited to have developed several models of distributed parameter systems for optimization of aeroelastic systems[37]. This later laid foundation for the development of distributed parameter control theory. Bailey and Hubbard[38] were among the first to demonstrate the active vibration damping of a cantilever beam using distributed parameter control logic, via Lyapunov's second method. This was extended to accommodate more general boundary conditions and non-uniform actuator spatial distributions by Burke and Hubbard[39]. The interesting aspect of this approach of treating the control as a distributed parameter is that there is no need for the discretizing the states to obtain a discretized model of the distributed parameter plant. The control partial differential equation is obtained 
directly along with its own boundary conditions.

However, the grace of this method is lost immediately owing to the fact that most of the real life applications use the discretized equations due to the collocated nature of the sensor outputs and actuator inputs (although smart material maybe represented as a continuum, the input voltages have to be applied at finite locations). Recently, there has been a lot of work in the area of distributed actuation. Fromherz and Jackson[40] implement a paper transport method, using precomputed analytical solution to the total force averaging problem which is posed as a constrained optimization problem. Hogg and Huberman[41] propose a heirarchical structure to be ideal for controlling smart matter. Therefore to control smart material, we go one step further and propose an adaptive aggregation scheme.

\section{A. Algorithm for Adaptive Discretization}

In the adaptive aggregation scheme,using physics based model, we populate the control influence matrix iteratively in accordance with a performance measure. This is done as described in figure 8. We start with a control influence matrix and evaluate the actuator position effectiveness by an actuator placement effectiveness measure. In the cantilever beam example presented in figures $9,10,11$, we use the measure proposed by Hamdan and Nayfeh[42]. This measure forms a matrix whose $i, j t h$ element approximates the influence of $j t h$ actuator on the $i t h$ mode of the system. The algorithm is shown to place actuator nodes at several locations along the length on a beam problem. The node insertion for each iteration is plotted in Fig. 10. Fig.11 shows the improvement of condition number of the control influence matrix of the beam problem with each node insertion. However, the node insertion shown in this simple example is not sufficient. The node insertion at each iteration is performed 
only at locations with largest controllability index. This results in what is called a greedy algorithm leading to insertion of nodes at the same locations each time. As a result there are an increasingly large number of nodes at sensitive locations. This issue is to be handled by a more uniform methodology of node insertion. This methodology becomes more important for distributed actuation in large structures as there is an increased need for sensitivity based aggregation in such applications. However, the practicality of the algorithm dictates to have it working along with a spatial discretization tool.

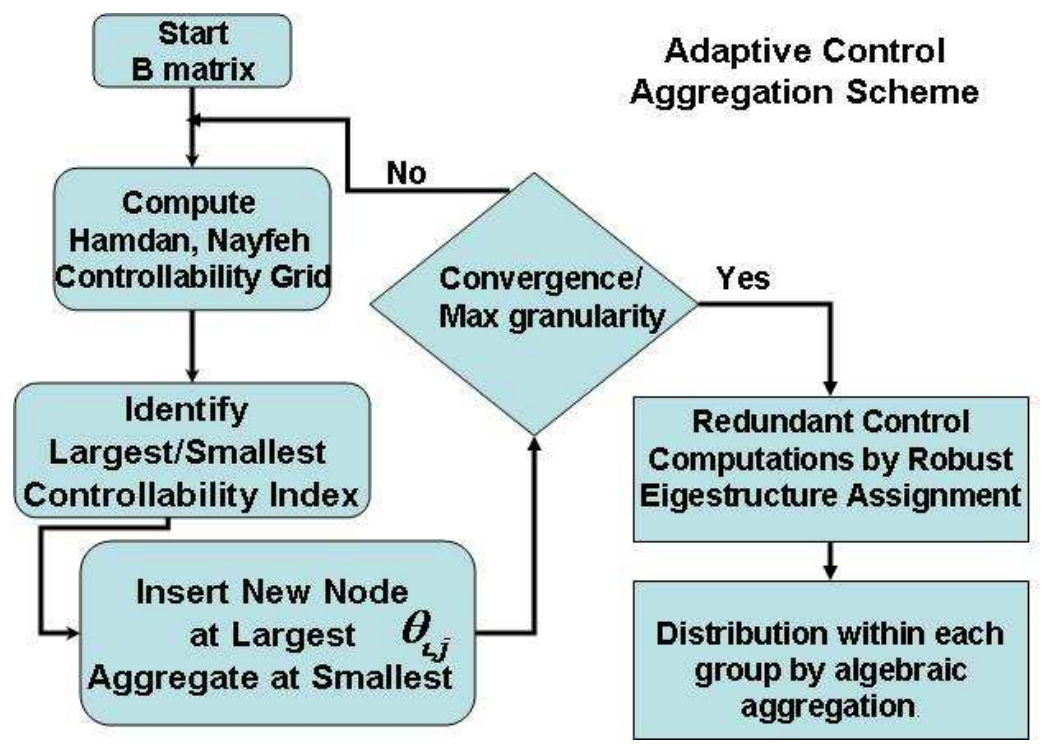

Fig. 9. Schematic of the adaptive aggregation scheme for hyper-redundant actuation

B. Application to the Morphing Wing

The above discretization procedure is applied to a finite element model of a novel morphing wing. The wing, shown in figure 12, consists of three twisting sections that change the wing twist profile, owing to the elastomeric skin that deforms on a 


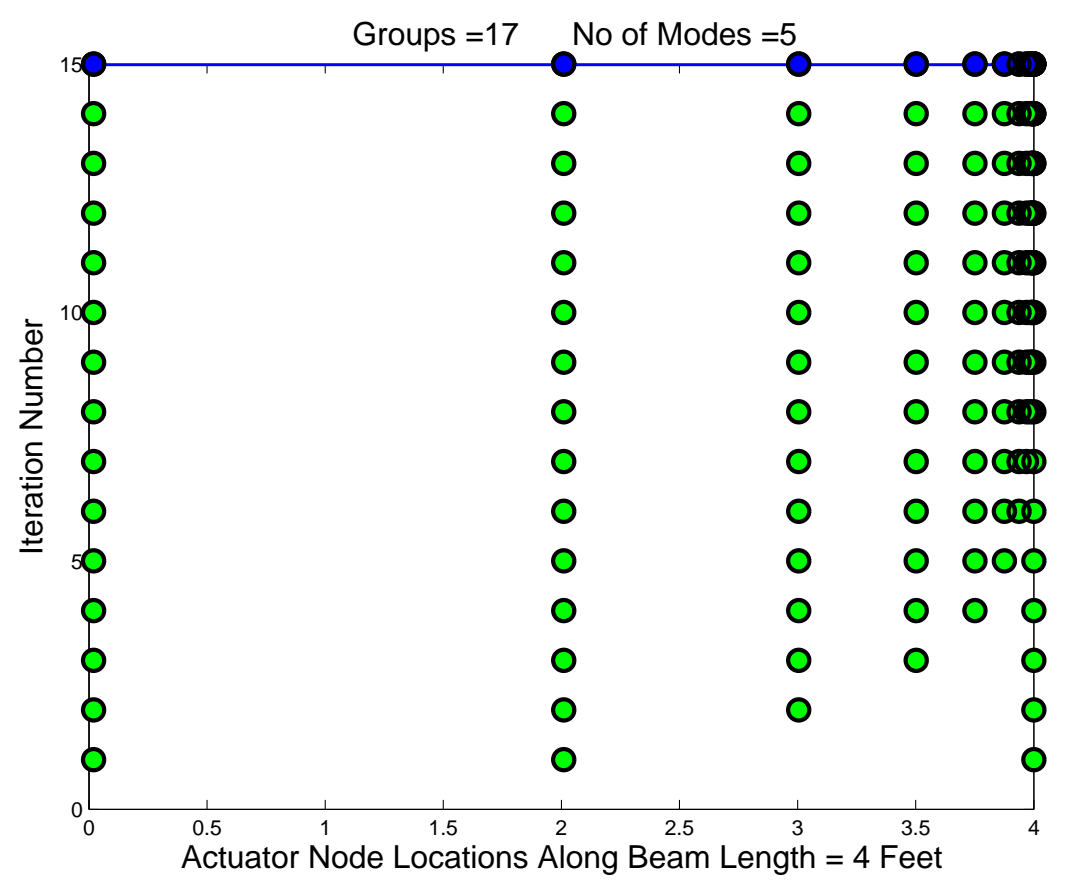

Fig. 10. Aggregation history with Hamdan Nayfeh Greedy strategy

flexible skeleton. An overly simplified model, with large model errors is being used to demonstrate the methodology. The actual structure of the wing is nonlinear due to several elements. Viscoelasticity, together with the coupled aerodynamics make the problem more complex. As a preliminary analysis, the wing is approximated by three members in torsion with varying geometric parameters (polar moment of inertia). The mass and stiffness matrices are computed from a three element finite element model of this structure (determined with with appropriate material properties). Five different force distributions are used as control inputs on the three elements and a second order mechanical system is constructed from the same, with four state variables and five control inputs. The open loop eigenvalues and close loop eigenvalues achieved are shown in table II, together with the condition numbers of the closed loop and the open loop modal matrix. Note that the desired closed loop modal matrix condition number is achieved exactly due to the partial eigenstructure assignment. 


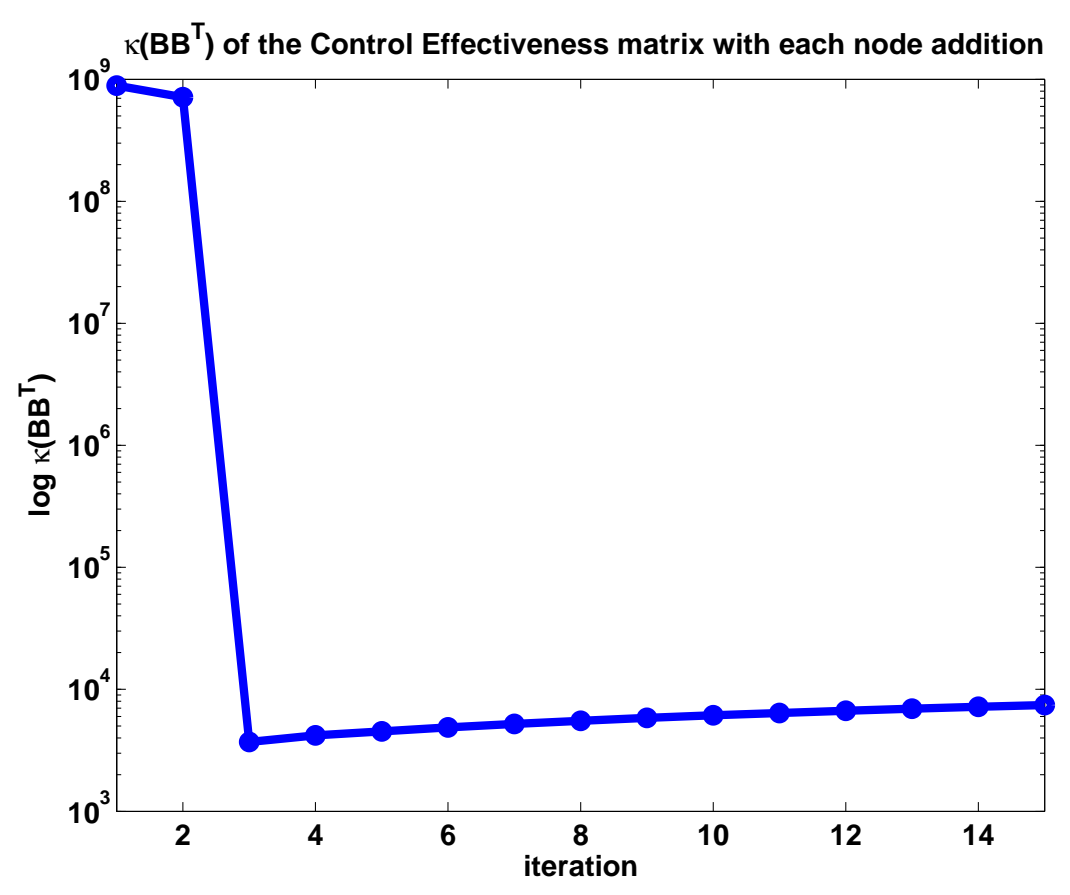

Fig. 11. Conditioning of the control influence matrix with mode addition

Clearly, we need a methodology to identify the unmodeled dynamics to compensate for the modeling errors that we have committed to this model. An implementation of this algorithm therefore should use an estimator of the eigen frequencies of the model error and try to compensate them to maintain stability and achieve the set performance goals. One way to do this is to have a sliding window and construct a linear model via adaptation and try to compensate for stability. Such an idea is discussed in the appendix. But the idea presented in the appendix is not suitable for a real time application due to several reasons. We may want to use a method to identify the frequencies from a sliding window of sampled data. The Eigensystem Realization Algorithm[43] is a potential candidate to extract the participating modes.

Thus, for this type of problems of hyper-redundant actuator arrays, which are poorly modeled in general, the discretization scheme, together with the robust redun- 
dant eigenstructure assignment is promising to be a simple and practical approach.

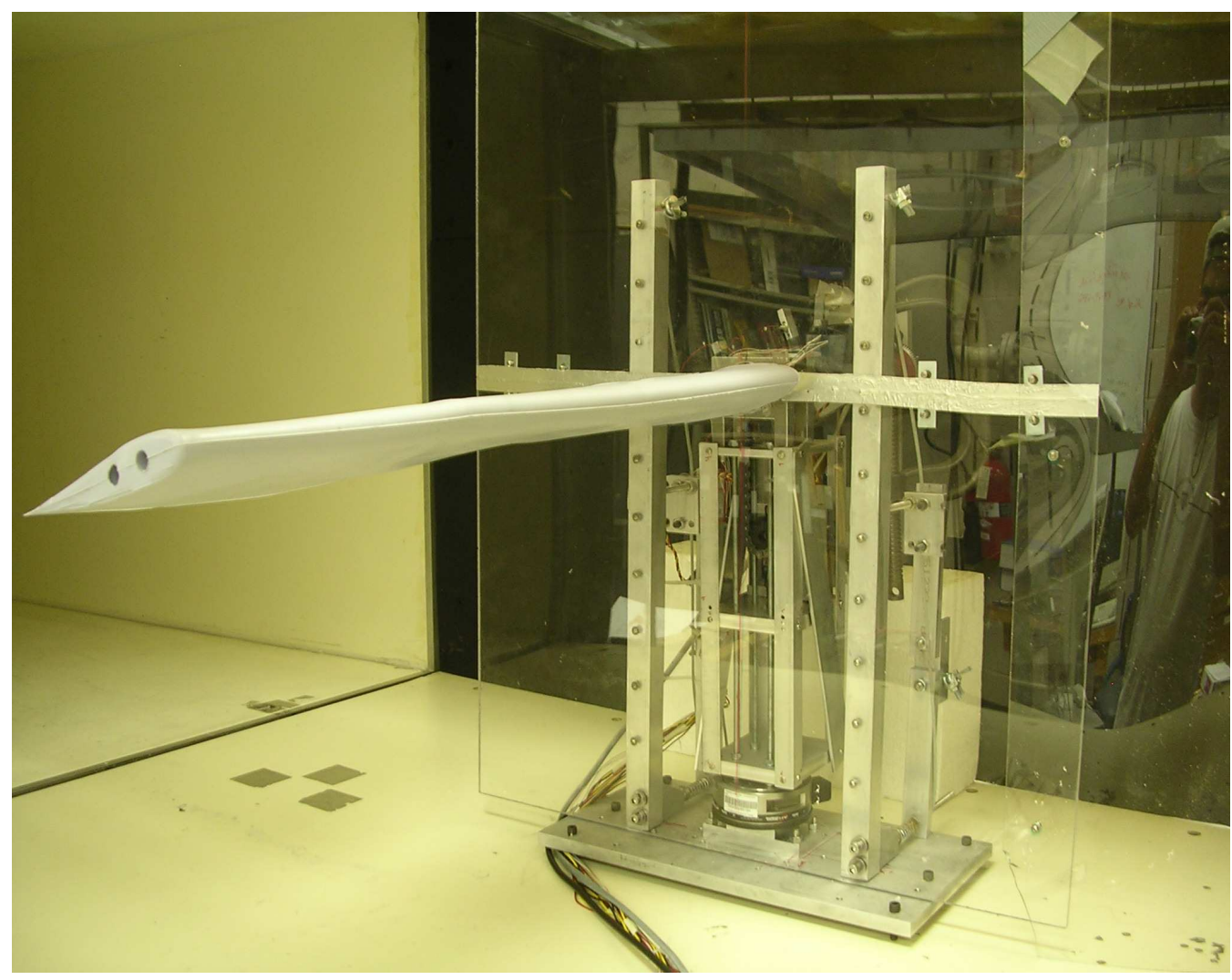

Fig. 12. Morphing Wing experiment 
Table II. Eigenvalues for the structural model of the morphing wing

\begin{tabular}{|c|c|c|}
\hline Openloop Eigenvalues & Target Eigenvalues & Closeloop Eigenvalues \\
\hline $0+163.3 \mathrm{i}$ & $-100+163.3 \mathrm{i}$ & $-100+163.3 \mathrm{i}$ \\
$0-163.3 \mathrm{i}$ & $-100-163.3 \mathrm{i}$ & $-100-163.3 \mathrm{i}$ \\
$247.21 \mathrm{i}$ & $-200+247.21 \mathrm{i}$ & $-200+247.21 \mathrm{i}$ \\
$-247.21 \mathrm{i}$ & $-200-247.21 \mathrm{i}$ & $-200-247.21 \mathrm{i}$ \\
$341.01 \mathrm{i}$ & $-300+341.01 \mathrm{i}$ & $-300+341.01 \mathrm{i}$ \\
$-341.01 \mathrm{i}$ & $-300-341.01 \mathrm{i}$ & $-300-341.01 \mathrm{i}$ \\
\hline Openloop Modal $\kappa(\Phi)$ & Desired Close Loop $\kappa(\Phi)$ & Achieved Cond. No. $\kappa(\Phi)$ \\
\hline 10.8555 & 2.3719 & 2.3719 \\
\hline
\end{tabular}




\section{CHAPTER VI}

\section{CONCLUSION}

A robust approach for the control of systems with greater number of inputs than the states of the plant model is presented in the thesis. The approach uses an extension to eigenstructure assignment algorithm, originally developed with a focus on under actuated systems. For second order mechanical systems, the partial eigenstructure assignment methodology is proposed. For real submodal target matrices, the position and velocity feedback gain calculation equations are given in a two step process. These results have been compared with traditional assignment approaches. The procedure is demonstrated on a robot with uncertain properties and robust tracking of reference trajectories is shown.

An important issue in redundancy management, coordination is discussed. A novel trained weighting approach is presented, which facilitates an online weighting of the control forces depending on the local controllability of individual actuator sets.

The methods proposed in the thesis are applied to hyper redundant actuator sets by an adaptive aggregation strategy, which helps bring down actuator dimensionality from infinity to finite inputs.

Directions for Future Research: It is felt that the redundancy in actuation can bring about some difference in systems with output feedback form. Further results in this direction have to be developed. The guaranteed stability region due to the Patel Toda theorem, is improved if we could construct a compensator based on discrete time data as indicated in the appendix. A strong theoretical foundation is recommended for the adaptive aggregation approach so as to extend the results for smaller orders 
of redundancy to hyper redundant actuation. Application of the above methodology to an experimental structure is also recommended in order to achieve a well desired closure between theory and practice. 


\section{REFERENCES}

[1] Nakamura, Y., Advanced Robotics : Redundancy and Optimization, Reading, MA: Addison-Wesley Publishing Company, 1991, pp. 105-282.

[2] Junkins, J. L., and Turner, J. T., Optimal Spacecraft Rotational Maneuvers, Studies in Astronautics, NewYork: Elsevier, 1986.

[3] Oh, H. S., and Vadali, S. R., "Feedback Control and Steering Laws for Spacecraft Using Single Gimbal Control Moment Gyros," The Journal of Astronautical Sciences, Vol. 39, No. 2, pp. 183-203, April-June 1991.

[4] Chirikjian,G. S., and Burdick, J. W., "Kinematically Optimal Hyper-Redundant Manipulator Configurations," IEEE Trans. Robot. Automat., Vol. 11, No. 6, pp. 794-806, December 1999.

[5] Nakamura, Y., and Ghodoussi, M., "Dynamics of Closed Link Robot Mechanisms with Nonredundant and Redundant Actuators," IEEE Transactions on Robotics and Automation, Vol. 5, No. 3, June 1989, pp. 294-302.

[6] Cheng, H., Yiu,Y. K., and Li,Z., "Dynamics and Control of Redundantly Actuated Parallel Manipulators," IEEE/ASME Transactions on Mechatronics, Vol. 8, No. 4, pp. 483-491, December 2003.

[7] Zheng,Y., and Qain,W. H., "Dynamic Force Distribution in Multifingered Grasping by Decomposition and Positive Combination," IEEE Transactions on Robotics, Vol. 21,No. 4, pp. 719-726, August 2005.

[8] Junkins, J. L., Akella, M. R., and Kurdila, A. J., "Adaptive Realization of Desired Constraint Stabilization Dynamics in the Control of Multibody Systems," Transactions of the Royal Society, Vol. 359, pp. 2231-2249, 2001. 
[9] Buffington,J. M. and Enns, D.F., "Lyapunov Stability Analysis of Daisy-Chain Control Allocation," Journal of Guidance Control and Dynamics, Vol. 19, No. 6, pp. 1226-1230, December 1996.

[10] Durham,W., C., "Computationally Efficient Control Allocation," Journal of Guidance Control and Dynamics, Vol. 24, No. 3,pp. 519-524, May - June 2001.

[11] Bodson,M., "Evaluation of Optimization Methods for Control Allocation," Journal of Guidance Control and Dynamics, Vol. 25, No. 4, pp. 703-711, July - August 2002.

[12] Singla, P., "A Multi-Resolution Approach for High Fidelity Modeling and Control Allocation in Large Scale Dynamical Systems," PhD Dissertation, Aerospace Engineering, Texas A\&M University, College Station, May 2006, pp. 232-269.

[13] Hughes,P. C., and Skelton,R. E., "Controllability and Observability of Linear Matrix-Second-Order Systems," Journal of Applied Mechanics, Vol. 47, pp. 415 - 420, June, 1980.

[14] Bedrossian,N. S., "Steering Law Design for Redundant Single Gimbal Control Moment Gyro Systems," M.S. Thesis, Mechanical Engineering, Massachusetts Institute of Technology, Cambridge, August 1987.

[15] Schaub, H., Vadali, S. R., and Junkins, J. L., "Feedback Control Law for Variable Speed Control Moment Gyroscopes," Journal of Astronautical Sciences, Vol. 46, No. 3, pp. 307-32, July-Sept., 1998.

[16] Tsui, C., C., Robust Control System Design : Advanced State Space Techniques, NewYork: Marcel Dekker, 2nd edition, 2004. 
[17] Wonham, W., M., "On the Pole Assignment of Multi-Input Controllable Linear Systems," IEEE Transactions on Automatic Control, Vol. AC-12, No. 6, pp. 660666, 1967.

[18] Srinathkumar, S., "Eigenvalue/Eigenvector Assignment Using Output Feedback," IEEE Transactions on Automatic Control, Vol. AC-23, No. 1, pp. 79-81, 1978.

[19] Srinathkumar, S., and Rhoten, R. P., "Eigenvalue/Eigenvector Assignment for Multivariable Systems," Electronics Letters, Vol. 11, No. 6,pp. 124-125, March 1975.

[20] Moore, B. C., "On the Flexibility Offered by State Feedback in Multivariable Systems Beyond Closed Loop Eigenvalue Assignments," IEEE Transactions on Automatic Control, Vol. AC-21, pp. 689-692, 1976.

[21] Sinswat, V., and Fallside, F., "Eigenvalue/Eigenvector Assignment by State Feedback," International Journal of Control, Vol. 26, No. 3, pp. 389-403, 1977.

[22] Kautsky, J., Nichols, N. K., and van Dooren, P., "Robust Pole Assignment in Linear State Feedback," International Journal of Control, Vol. 41, No. 5, pp. 1129$1155,1985$.

[23] Bhattacharya, S. P., Desouza, E., "Pole Assignment via Sylvester's Equations," Systems and Control Letters, Vol. 1, No. 4, pp. 261 - 263, 1982.

[24] Calvin, R. K., and Bhattacharya, S. P., "Robust and Well-Conditioned Eigenstructure Via Sylvester's Equation," Journal of Optimal Control Applications and Methods, Vol. 4, pp. 205 - 212, 1983. 
[25] Rew, D. W., Junkins, J. L., and Juang, J. N., "Robust Eigenstructure Assignment by a Projection Method: Applications Using Multiple Optimization Criteria," Journal of Guidance Control and Dynamics, Vol. 12, No. 3, pp. 396 403, 1989.

[26] Juang, J. N., Lim, K. B., and Junkins, J. L., "Robust Eigensystem Assignment for Flexible Structures," Journal of Guidance Control and Dynamics, Vol. 12, No. 3, pp. 381 - 387, 1989.

[27] Juang, J., N. and Maghami, M., "Robust Eigensystem Assignment for Second Order Dynamic Systems," in Mechanics and Control of Large Flexible Structures, John L. Junkins Ed., AIAA: Washington, DC, AIAA, 1990,Progress in Astronautics and Aeronautics, pp. 373-387.

[28] Kim, Y.,Kim, H. S., and J. L. Junkins., "Eigenstructure Assignment Algoritm for Mechanical Second-Order Systems," Journal of Guidance Control and Dynamics, Vol. 22, No. 5, pp. 729-31, 1999.

[29] Junkins, J. L., and Kim, Y., Introduction to the Dynamics and Control of Flexible Spacecraft Structures, AIAA Education Series, AIAA: Washington, DC, 1993.

[30] Patel, R. V., and Toda, M., "Quantitative Measures of Robustness for Multivariable Systems," in Proc Joint Automatic Control Conference, AIAA, San Francisco, CA, 1980, TP-8A, Vol. 1.

[31] Bauer, F. L., and Fike, C. T., "Norms and Exclusion Theorems," Numerische Mathematique, Vol. 2.,pp. 137-141,1960.

[32] Junkins, J. L., and Kim, Y., "Robust Eigensystem Assignment for Second Order 
Dynamic Systems," in Teleoperation and Robotics in Space, S. B. Skaar and C. F. Ruoff, Ed.,Washington, DC, AIAA, 1994, Progress in Astronautics and Aeronautics, pp. 315-349.

[33] Threfethen, L., N.,and Bau, D.,Numerical Linear Algebra, Philadelphia, PA: SIAM, 1997.

[34] Golub,G. H., and VanLoan,C. F., Matrix Computations, Baltimore,MD: Johns Hopkins University Press, 3rd edition, 1996.

[35] Wang, P. K. C., "Stability Analysis of Elastic and Aeroelastic Systems via Lyapunov's Direct Method," Journal of the Franklin Institute, Vol. 281, pp. 51-72, 1966.

[36] Wang, P. K. C., "Stability Analysis of a Simplified Flexible Vehicle via Lyapunov's Direct Method," AIAA Journal, Vol. 3, No. 9,pp. 1764-1766, 1965.

[37] Wu, Y. T., Chwang, A. T., and Wang, P. K. C., "Optimization Problems in Hydrofoil Propulsion," in Optimization and Stability Problems in Continuum Mechanics, P. K. C. Wang, Ed., New York: Springer Verlag, 1971.

[38] Bailey, T, and Hubbard, J. E. Jr., "Distributed Piezoelectric-Polymer Active Vibration Control of a Cantilever Beam," Journal of Guidance Control and Dynamics, Vol. 8, pp. 605 - 611, 1985.

[39] Burke,S. E., and Hubbard,J. E. Jr., "Distributed Actuator Control Design for Flexible Beams," Automatica, Vol. 24, No. 5, pp. 619 - 627, 1988.

[40] Fromherz, M. P. J., and Jackson, .W. B., "Force Allocation in a Large Scale Distributed Active Surface," IEEE Transations on Control Systems Technology, Vol. 11., No. 5, pp. 641-655, 2003. 
[41] Hogg, T., and Huberman, B. A., "Controlling Smart Matter," Smart Mater. Struct, Vol. 7, R1-R14, 1998.

[42] Hamdan, A. M. A., and Nayfeh, A. H., "Measures of Modal Controllability and Observability of First and Second - Order Linear Systems," Journal of Guidance Control and Dynamics, Vol. 12, No. 3, pp. 421 - 427, 1989.

[43] Pappa, R. S. and Juang, J. N., "Some Experiences with the Eigensystem Realization Algorithm," Sound and Vibration, Vol. 22, No. 1, pp. 30-35, 1988.

[44] Kristic, M., Kanellakopoulos, I., Kokotovic, P., Nonlinear and Adaptive Control Design, NewYork: John Wiley and Sons, 1995.

[45] Iounnou, P., and Sun, J., Robust Adaptive Control, NewYork: Prentice Hall, 1997. 


\section{APPENDIX A}

\section{ASSIGNABILITY OF EIGENSTRUCTURE}

In this appendix, we summarize the result of Srinathkumar [18], about the assignable components in the closed loop modal matrix. This is stated in the following theorem. The original theorem stated in [18] has been modified and the full state feedback version is presented for clarity. Extension to output feedback is straightforward. Let us consider the Linear system with some partitions, before we state the theorem,

$$
\dot{x}=A x+B u=\left[\begin{array}{cc}
A_{11} & A_{12} \\
A_{21} & A_{22}
\end{array}\right] x+\left[\begin{array}{c}
B_{1} \\
B_{2}
\end{array}\right] u
$$

$B \in \Re^{n \times m}, m<n, A \in \Re^{n \times n}, x \in \Re^{n}, u \in \Re^{m}$, with $B_{1} \in \Re^{m \times m}$ nonsingular.

Theorem A.1 (Srinathkumar and Rhoten[18]) Given a linear system $(A, B)$, of the form equation A.1, and a state feedback control law, $u=K x$, only $m$ entries in each closed loop mode may be arbitrarily assigned.

Proof The closed loop eigenvalue problem can be written as

$$
(A+B K) u_{i}=\lambda_{i} u_{i}
$$

It would be convenient to introduce the partition of the eigenvector, $u_{i}=\left[z_{i}^{T}, w_{i}^{T}\right]^{T}$. With this partition, the closed loop eigenvalue problem becomes,

$$
\left[\begin{array}{cc}
A_{11}+B_{1} K_{1} & A_{12}+B_{1} K_{2} \\
A_{21}+B_{2} K_{1} & A_{22}+B_{2} K_{2}
\end{array}\right]\left(\begin{array}{c}
z_{i} \\
w_{i}
\end{array}\right)=\lambda_{i}\left(\begin{array}{c}
z_{i} \\
w_{i}
\end{array}\right)
$$


Eliminating the feedback gains, we get

$$
\begin{gathered}
\left(\left[A_{11}^{T}, A_{12}^{T}\right]^{T}+B_{1} K\right) u=\lambda z \\
\left(\lambda I_{n-m}-F\right) w=(G+\lambda S) z
\end{gathered}
$$

where $G=A_{21}-S A_{11}, F=A_{22}-S A_{12}$ and $S=B_{2} B_{1}^{-1}$. Clearly, equation A.5, is a set of $n-m$ constraints. Therefore, $m$ components, of $u_{i}$, namely, $z_{i}$ can be assigned arbitrarily. 


\section{APPENDIX B}

\section{ADAPTATION TO ACCOMMODATE UNMODELED DYNAMICS}

In this appendix, we present a technique of construction of a linear model from the input and state information. This could be applied for the identification of a dynamic model, whose states are the unmodeled plant dynamics, to be used for compensation by the control law. Let us discuss the methodology and then apply it to a two link manipulator example.

Before talking about control, let us talk a little bit about a possible way of controlling a dynamical system without the knowledge of its mathematical model. Consider the nonlinear system,

$$
\dot{x}=f(x, u)
$$

for a given reference trajectory, $q_{r e f}(t)$, let there exist a control to make the tracking error go to zero. Assuming the trajectory and reference are continuously differentiable, the error dynamics is continuous and is locally linearizable. Thus we assume the validity of the linearization about a nominal trajectory.

Now, let us consider the linearized system,

$$
\dot{x}=\left.\frac{\partial f}{\partial x}\right|_{x_{n o m}, u_{n o m}} x+\left.\frac{\partial f}{\partial u}\right|_{x_{n o m}, u_{n o m}} u+f\left(x_{\text {nominal }}, u_{\text {nominal }}\right)
$$

The error dynamics therefore could be assumed to be linear in the local domain $D \in \Re_{\text {someneighborhood. The linear dynamics can thus be written as }}$

$$
\dot{e}=A_{p} e+B_{p} u+d_{p}
$$


in some domain $D$. This can therefore be identified in that domain by standard adaptive control methods. Following the standard adaptive control regimen $[44,45]$, we construct the Lyapunov functional.

$$
V\left(\tilde{A}_{p}, \tilde{B}_{p}, \tilde{d}_{p}, \epsilon_{1}\right)=\frac{1}{2} \epsilon_{1}^{T} P \epsilon_{1}+\operatorname{tr}\left\{\frac{\tilde{A}_{p}^{T} P \tilde{A}_{p}}{2 \gamma_{1}}\right\}+\operatorname{tr}\left\{\frac{\tilde{B}_{p}^{T} P \tilde{B}_{p}}{2 \gamma_{2}}\right\}+\frac{1}{2} \tilde{d}_{p}^{T} P \tilde{d}_{p}
$$

giving us the identification laws

$$
\begin{gathered}
\dot{\hat{A}}_{p}=\gamma_{1} \epsilon_{1} e^{T} \\
\dot{\hat{B}}_{p}=\gamma_{2} \epsilon_{1} u^{T} \\
\dot{\hat{d}}_{p}=\gamma_{3} \epsilon_{1}
\end{gathered}
$$

With these online parameter estimates, we can construct an instantaneous linearized system, which represents the error dynamics, along a reference trajectory of the unknown system. This is systematically done as follows.

- Consider the region of interest in the state space and construct the reference trajectory.

- Split the trajectory in to intervals.

- This can be done by considering small intervals of time.

- Owing to the state continuity, small time intervals lead to small domains in the state space making the linear approximation valid. This interval thus becomes a design variable.

- Extract the state variable and compute the tracking error from the reference trajectory.

- Integrate the identification equations along with the dynamics. 
- Design an appropriate control law to regulate the error dynamics. (Use certainty equivalence left right and center !!!)

Example Application Having developed a methodology for model error compensation, we would now like to apply it to the mathematical model of a two link open chain manipulator(the rigid cousin of example 4.6 in Junkins and Kim [29], shown in figure 15). Over a span of 20 seconds, 10 intervals of 2 seconds each were considered for realizing an equivalent linear model via the proposed methodology. Since the control influence matrix, $B$ is known, we opt not to learn it via adaptation.

However there is a problem that we have and that is that the control torque inputs to the system affect only the velocity states and do not affect the position states. The danger in this scenario is clearly seen from the following identified system equations (in first order state space form)

$$
\left[\begin{array}{c}
\dot{e_{1}} \\
\dot{e_{2}}
\end{array}\right]=\left(\begin{array}{cc}
\hat{A}_{p 11} & \hat{A}_{p 12} \\
\hat{A}_{p 21} & \hat{A}_{p 22}
\end{array}\right)\left(\begin{array}{c}
e_{1} \\
e_{2}
\end{array}\right)+\left(\begin{array}{c}
0_{2 \times 2} \\
I_{2 \times 2}
\end{array}\right)\left(\begin{array}{c}
\tau_{1} \\
\tau_{2}
\end{array}\right)+\left(\begin{array}{c}
\hat{d}_{1} \\
\hat{d}_{2}
\end{array}\right)
$$

Clearly the equation B.8 shows that the unforced kinematics part of the system, is experiencing disturbance from $\hat{d}_{1}$. This disturbance has to be rejected. We could use the ES assignment approach, to reject the disturbance by making the target close loop poles farther away from the imaginary axis. But we choose to use another nice nonlinear control methodology, Backstepping[44]. Let us now derive a control law for this system via backstepping and implement the same along with the adaptive linearized identification methodology.

This has been implemented on the nonlinear robot model in tandem with the linearized identification strategy in small intervals of 2 seconds at a time. The results 
are shown in the figures 13,14 . We emphasize the elegance of this method. Nowhere have the physics based equations been used in the entire control law development. It is assumed that the plant model is completely unknown and only the state and the control information is available to the designer.

Thus this methodology can be used to construct a linear model over a sliding window of continuous time state information.

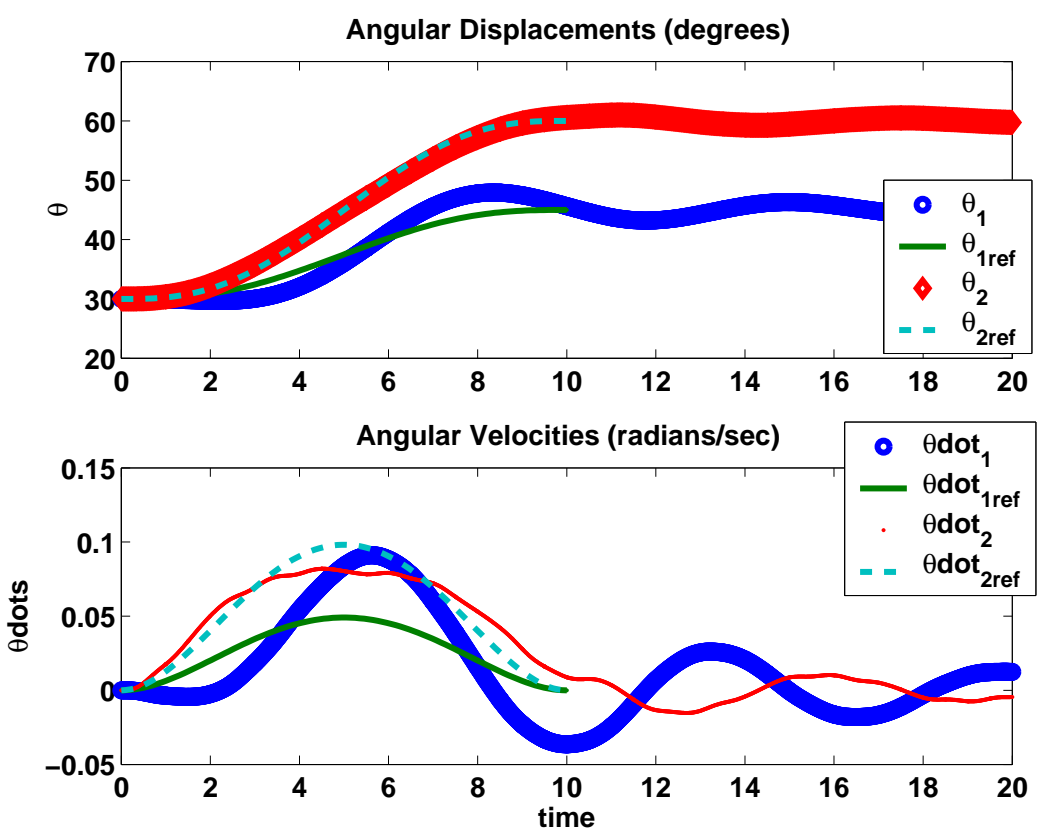

Fig. 13. Tracking control law with adaptive local linearization scheme 


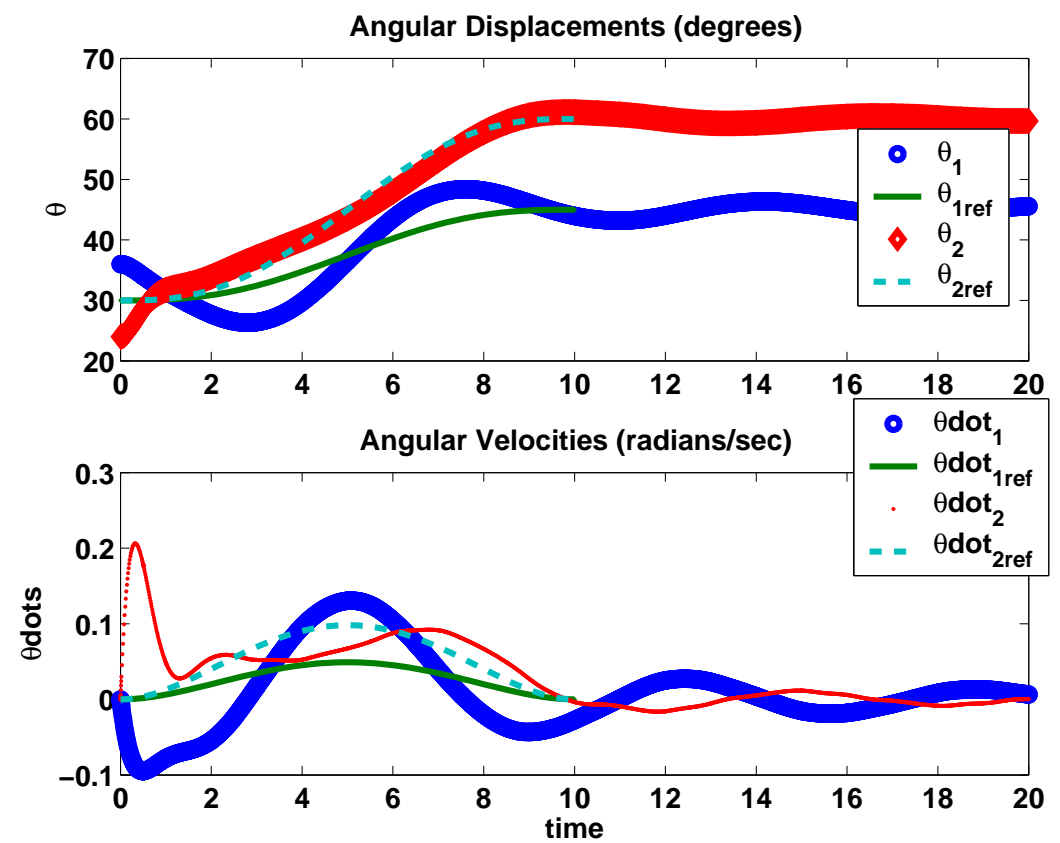

Fig. 14. Tracking control with $20 \%$ perturbed initial conditions

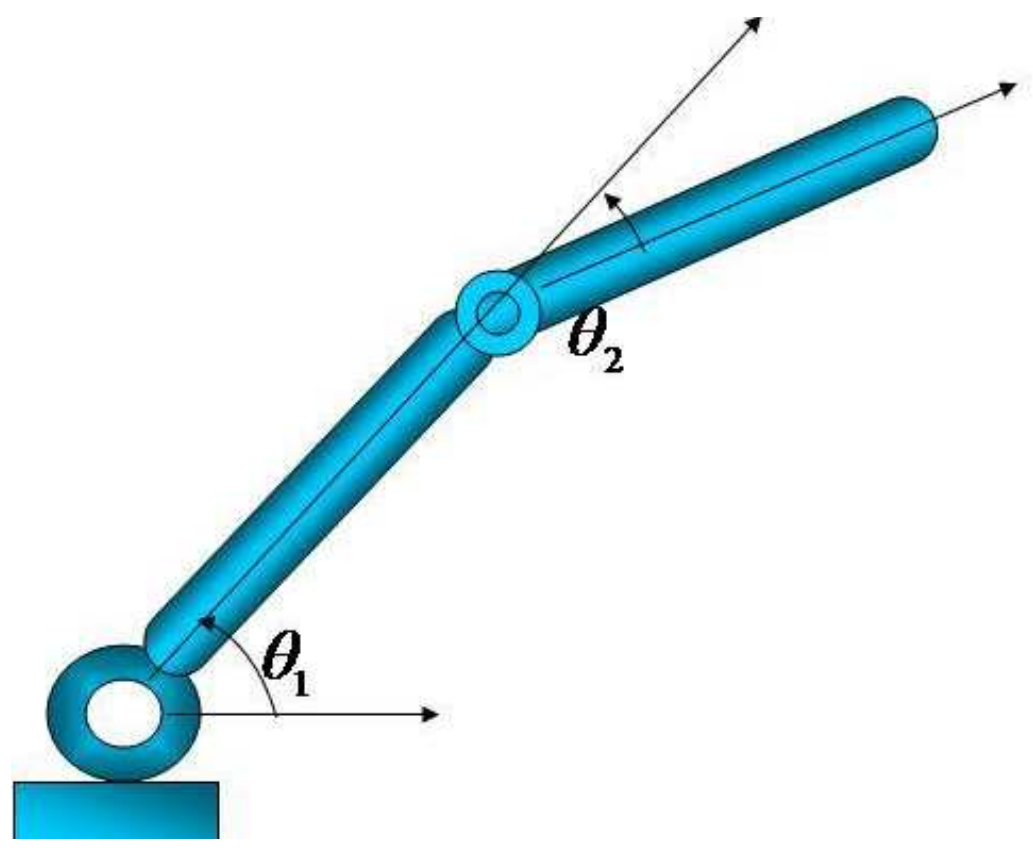

Fig. 15. Schematic of the two link rigid manipulator 


\section{VITA}

Manoranjan Majji received his Bachelor of Engineering (Honors) in mechanical engineering from Birla Institute of Technology and Science, Pilani, India in 2003. His research interests include robust redundant actuation and sensing, estimation, optimization, adaptive and distributed control applications. He will continue his graduate education in pursuit of a doctoral degree starting in the summer of 2006 . He is a student member of AIAA, SIAM.

Contact Address: 608, HRBB, 3141, TAMU; College Station, TX 77843-3141 